\title{
Local Decodability of the Burrows-Wheeler Transform*
}

\author{
Sandip Sinha \\ sandip@cs.columbia.edu \\ Columbia University \\ New York, NY, USA
}

\author{
Omri Weinstein \\ omri@cs.columbia.edu \\ Columbia University \\ New York, NY, USA
}

\begin{abstract}
The Burrows-Wheeler Transform (BWT) is among the most influential discoveries in text compression and DNA storage. It is a reversible preprocessing step that rearranges an $n$-letter string into runs of identical characters (by exploiting context regularities), resulting in highly compressible strings, and is the basis of the bzip compression program. Alas, the decoding process of BWT is inherently sequential and requires $\Omega(n)$ time even to retrieve a single character.

We study the succinct data structure problem of locally decoding short substrings of a given text under its compressed BWT, i.e., with small additive redundancy $r$ over the Move-To-Front (bzip) compression. The celebrated BWT-based FM-index (FOCS '00), as well as other related literature, yield a trade-off of $r=\tilde{O}(n / \sqrt{t})$ bits, when a single character is to be decoded in $O(t)$ time. We give a near-quadratic improvement $r=\tilde{O}(n \lg (t) / t)$. As a by-product, we obtain an exponential (in $t$ ) improvement on the redundancy of the FM-index for counting pattern-matches on compressed text. In the interesting regime where the text compresses to $o(n)$ (say, $n / \operatorname{polylg}(n))$ bits, these results provide an $\exp (t)$ overall space reduction. For the local decoding problem of BWT, we also prove an $\Omega\left(n / t^{2}\right)$ cell-probe lower bound for "symmetric" data structures.

We achieve our main result by designing a compressed partialsums (Rank) data structure over BWT. The key component is a locally-decodable Move-to-Front (MTF) code: with only $O(1)$ extra bits per block of length $n^{\Omega(1)}$, the decoding time of a single character can be decreased from $\Omega(n)$ to $O(\lg n)$. This result is of independent interest in algorithmic information theory.
\end{abstract}

\section{CCS CONCEPTS}

- Mathematics of computing $\rightarrow$ Information theory; - Theory of computation $\rightarrow$ Data structures design and analysis.

\section{KEYWORDS}

pattern matching, burrows-wheeler transform, local decoding, cellprobe model, succinct data structures, text compression

\footnotetext{
* This work was supported by NSF awards CCF-1563155, CCF-1420349, CCF-1617955, CCF-1740833, CCF-1421161, CCF-1714818, CAREER award CCF-1844887 and Simons Foundation (\#491119).

Permission to make digital or hard copies of all or part of this work for personal or classroom use is granted without fee provided that copies are not made or distributed for profit or commercial advantage and that copies bear this notice and the full citation on the first page. Copyrights for components of this work owned by others than ACM must be honored. Abstracting with credit is permitted. To copy otherwise, or republish, to post on servers or to redistribute to lists, requires prior specific permission and/or a fee. Request permissions from permissions@acm.org.

STOC '19, June 23-26, 2019, Phoenix, AZ, USA

(C) 2019 Association for Computing Machinery.

ACM ISBN 978-1-4503-6705-9/19/06 . .\$15.00

https://doi.org/10.1145/3313276.3316317
}

\section{ACM Reference Format:}

Sandip Sinha and Omri Weinstein. 2019. Local Decodability of the BurrowsWheeler Transform. In Proceedings of the 51st Annual ACM SIGACT Symposium on the Theory of Computing (STOC '19), fune 23-26, 2019, Phoenix, AZ, USA. ACM, New York, NY, USA, 12 pages. https://doi.org/10.1145/3313276. 3316317

\section{INTRODUCTION}

Exploiting text regularities for data compression is an enterprise that received a tremendous amount of attention in the past several decades, driven by large-scale digital storage. Motivated by this question, in 1994 Burrows and Wheeler [6] proposed a preprocessing step that 'tends' to rearrange strings into more 'compressible form': Given $x \in \Sigma^{n}$, generate the $n \times n$ matrix whose rows are all cyclic shifts of $x$, sort these rows lexicographically, and output the last column $L$ of the matrix. The string $L$ is called the BurrowsWheeler Transform (BWT) of $x$. Note that $L$ is a permutation of the original string $x$, as each row of the sorted matrix is still a unique cyclic shift. The main observation is that, in the permuted string $L$, characters with identical context ${ }^{1}$ appear consecutively, hence if individual characters in the original text $x$ tend to be predicted by a reasonably small context (e.g., as in English texts or bioinformatics [1]), then the string $L:=\mathrm{BWT}(x)$ will exhibit local similarity. That is, identical symbols will tend to recur at close vicinity. This property suggests a natural way to compress $L$, using a relative recency coding method, whereby each symbol in $L$ is replaced by the number of distinct symbols that appeared since its last occurrence. Indeed, since in $L$ symbols have local similarity, i.e., tend to recur at close vicinity, we expect the output string to consist mainly of small integers (and in particular, 0-runs) and hence be much cheaper to describe. This relative encoding, known as the Moveto-Front transform [3], followed by run-length coding of 0-runs and an arithmetic coding stage (henceforth denoted RLX $(L)$ ), forms the basis for the widespread bzip2 program [36]. The RLX ("bzip") compression benchmark was justified both theoretically and empirically $[9,20,24]$, where among other properties, it was shown to approximately converge to any finite-order empirical entropy $H_{k}$ (see Section 2.3 for the formal definitions).

Remarkably, the Burrows-Wheeler transform of a string is invertible. The crux of the decoding process is the fact that the transform preserves the order of occurrences (a.k.a rank) of identical symbols in both the first column and last column $(L)$ of the BWT matrix. This crucial fact facilitates an iterative decoding process, whereby, given the decoded position of $x_{i+1}$ in $L$, one can decode the previous character $x_{i}$ using $O(|\Sigma|)$ RANK $^{2}$ queries to $L$ (see Section 2.1.1 for the formal decoding algorithm). Alas, this decoding process is

\footnotetext{
${ }^{1}$ The $k$-context of a symbol $x_{i}$ in $x$ is the $k$ consecutive symbols that precede it. ${ }^{2}$ The RANK of a character $x_{i} \in x$ is the number of occurrences of identical symbols in $x$ preceding it: $\operatorname{RANK}(x, i):=\left|\left\{j \leq i: x_{j}=x_{i}\right\}\right|$. See Section 2 .
} 
inherently sequential, and therefore requires $\Omega(n)$ time to decode even a single index of $x[21,25]$. In fact, no sub-linear decoding algorithm for $x_{i}$ is known even if $L$ is stored in uncompressed form.

This is an obvious drawback of the Burrows-Wheeler transform, as many storage applications, such as genetic sequencing and alignment, need local searching capabilities inside the compressed database [30]. For example, if $x^{1}, x^{2}, \ldots, x^{m} \in \Sigma^{n}$ is a collection of $m$ files with very similar contexts, e.g., DNA sequences, then we expect $\left|\operatorname{RLX}\left(L_{x^{1} \circ x^{2} \circ \ldots \circ x^{m}}\right)\right| \ll \sum_{j=1}^{m}\left|\operatorname{RLX}\left(L_{x^{j}}\right)\right|$, but jointly compressing the files would have a major drawback - When the application needs to retrieve only a single file $x^{j}$, it will need to spend $\Omega(n \cdot m)$ $\mathrm{I} / \mathrm{Os}$ (instead of $O(n))$ to invert BWT $\left(x^{1} \circ x^{2} \circ \ldots \circ x^{m}\right)$. The main question we address in this paper is, whether small additive space redundancy (over the compressed BWT string $(|\mathrm{RLX}(\mathrm{BWT}(x))|)$ can be used to reduce the decoding time of a single character (or a short substring) of the original text, in the word-RAM model:

Problem 1. What is the least amount of space redundancy $r=r(t)$ needed beyond the compressed $\mathrm{BWT}(x)$ string, so that each coordinate $x_{i}$ can be decoded in time $t$ ?

Since $|\operatorname{RLX}(\operatorname{BWT}(x))|$ approaches any finite-order empirical entropy $H_{k}(x)$ (see Section 2.3), this data structure problem can be viewed as the succinct dictionary ${ }^{3}$ problem under infinite-order entropy space benchmark. A long line of work has been devoted to succinct dictionaries in the context-free regime, i.e., when the information theoretic space benchmark is the zeroth-order empirical entropy $H_{0}(x):=\sum_{c \in \Sigma} n_{c} \lg \frac{n}{n_{c}}$ of marginal frequencies (e.g., $[18,31,32]$ to mention a few). However, as discussed below, much less is known about the best possible trade-off under higher-order entropy benchmarks which take context (i.e., correlation between $x_{i}$ 's) into account.

A related but incomparable data structure problem to Problem 1, is that of compressed pattern-matching in strings, a.k.a full-text indexing, where the goal is to succinctly represent a text in compressed form as before, so that all $o c c(p)$ occurrences of a pattern $p \in \Sigma^{\ell}$ in $x$ can be reported, or more modestly, counted, in near-optimal time $O(\ell+o c c(p))$. The celebrated BWT-based compressed text index of Ferragina and Manzini [13], commonly known as the FM-index, achieves the latter task using $|\operatorname{RLX}(L)|+O(n / \lg n)$ bits of space ${ }^{4}$, and is one of the most prominent tools in pattern matching for bioinformatics and text applications (see [30] and references therein). The core of the FM-index is a compressed data structure that computes RANK queries over the (compressed) BWT string $L$ in constant time and redundancy $r=O(n / \lg n)$ bits, and more generally, in time $t^{\prime}$ and redundancy $r=\Theta\left(n /\left(t^{\prime} \lg n\right)\right)$. Their data structure, combined with simple "marking index" ${ }^{5}$ of the BWT permutation on blocks of length $t$, yields a solution to Problem 1 with overall redundancy $\tilde{O}\left(n / t+n / t^{\prime}\right)$ and time $O\left(t \cdot t^{\prime}\right)$ (as simulating each sequential step of the BWT decoder requires $O(1)$ rank queries, and there are $t$ coordinates per block). In other words, if the desired decoding time of a coordinate is $t$, [13] gives redundancy $r=\tilde{O}(n / \sqrt{t})$ over RLX. In

\footnotetext{
${ }^{3}$ For a string $x \in \Sigma^{n}$ and an index $i \in[n]$, Dictionary $(x, i)$ returns $x_{i}$, the $i^{t h}$ character in $x$.

${ }^{4}$ For reporting queries, [13] requires significantly larger space $|\mathrm{RLX}(L)| \cdot \lg ^{\varepsilon} n+$ $O\left(n / \lg ^{1-\varepsilon} n\right)$ for some $\varepsilon \in(0,1)$.

${ }^{5}$ I.e., recording shortcut pointers to the location of $x_{i}$ in $L$ for every block $i \in[j$. $t], j \in[n / t]$, see Section 3 .
}

fact, even for randomized succinct dictionaries approaching $H_{k}(x)$ bits of space, the best known trade-off is $r=\tilde{\Theta}(n / \sqrt{t})$ : Dutta et al. [8] gave a randomized data structure with space $(1+\varepsilon) \tilde{H}_{k}(x)^{6}$, and expected decoding time $\Theta\left(1 / \varepsilon^{2}\right)$ to retrieve each $x_{i}$. Our first main result is a near-quadratic improvement over this trade-off for Problem 1:

Theorem 1 (Local Decoding of BWT, Informal). For any $t$ and any string $x \in \Sigma^{n}$ (with $|\Sigma|=O(1)$ ), there is a succinct data structure that stores $|\operatorname{RLX}(\operatorname{BWT}(x))|+\tilde{O}\left(\frac{n \lg t}{t}\right)+n^{0.9}$ bits of space, so that each coordinate $x_{i}$ can be retrieved in $O(t)$ time, in the word-RAM model with word size $w=\Theta(\lg n)$.

Our data structure directly implies that a contiguous substring of size $\ell$ of $x$ can be decoded in time $O(t+\ell \cdot \lg t)$ without increasing the space redundancy. It is noteworthy that achieving a linear trade-off as above between time and redundancy with respect to zeroth order entropy $\left(H_{0}(x)\right)$ is trivial, by dividing $x$ into $n / t$ blocks of length $t$, and compressing each block using Huffman (or arithmetic) codes, as this solution would lose at most 1 bit per block. This solution completely fails with respect to higher-order entropy benchmarks $H_{k}$ (in fact, even against $H_{1}$ ), since in the presence of contexts, the loss in compressing each block separately can be arbitrarily large (e.g., $H_{0}\left((a b)^{n / 2}\right)=n$ but $H_{1}\left((a b)^{n / 2}\right)=\lg n$ ). This example illustrates the qualitative difference between the Dictionary problem in the independent vs. correlated setting. In fact, we prove a complementary cell-probe lower bound of $r \geq \Omega\left(n / t^{2}\right)$ on Problem 1 for "symmetric" data structures, which further decode the dispositions of any $x_{i}$ in $L=\operatorname{BWT}(x)$ and vice versa, in time $O(t)$ (see Theorem 3.2 below). While removing this (natural) restriction remains an interesting open question, this result provides a significant first step in understanding the cell-probe complexity of Problem 1; more on this below.

Our second main result, which is a by-product of our central data structure, is an exponential improvement (in $t$ ) on the redundancy of the FM-index for compressed pattern-matching counting (throughout the paper, we assume $|\Sigma|=O(1)$ ):

Theorem 2. There is a small constant $\delta>0$ such that for any $x \in \Sigma^{n}$ and any $t \leq \delta \lg n$, there is a compressed index using $|\operatorname{RLX}(\operatorname{BWT}(x))|+$ $n \lg n / 2^{t}+n^{1-\Omega_{\delta}(1)}$ bits of space, counting the number of occurrences of any pattern $p \in \Sigma^{\ell}$ in time $O(t \ell)$.

To the best of our knowledge, Theorem 2 provides the first compressed text index for pattern-matching counting queries, that can provably go below the $\Omega(n / \lg n)$ space barrier while maintaining near-optimal query time. In particular, it implies that at the modest increase of query time by a $O(\lg \lg n)$ factor, any $n /$ poly $\lg n$ redundancy is achievable. In the interesting setting of compressed pattern-matching, where the text compresses to $o(n)$ bits (e.g., $H_{k}(x)=n / \lg ^{O(1)} n$ ), this result provides an exponential (in $t$ ) overall (i.e., multiplicative) space reduction over the FM-index. Compressed string-matching in the $o(1)$ per-bit entropy regime was advocated in the seminal work of Farach and Thorup, see [10] and references therein. For reporting queries, we obtain a quadratic improvement over the FM-index, similar to Theorem 1. For $x \in \Sigma^{n}$ and a pattern

${ }^{6}$ Here, $\tilde{H}_{k}$ denotes the Lempel-Ziv [43] codeword length $|\operatorname{LZ78}(x)|$, i.e., $\tilde{H}_{k}(x) \approx$ $H_{k}(x)+\Theta(n / \lg n)$. 
$p \in \Sigma^{\ell}$, let $o c c(p)$ be the number of occurrences of $p$ as a contiguous substring of $x$.

Theorem 3. Fix a string $x \in \Sigma^{n}$. For any $t$, there is a succinct data structure that, given a pattern $p \in \Sigma^{\ell}$, reports the starting positions of the occ $(p)$ occurrences of $p$ in $x$ in time $O(t \cdot o c c(p)+\ell \cdot \lg t)$, using at most $|\operatorname{RLX}(\mathrm{BWT}(x))|+O\left(\frac{n \lg n \lg t}{t}\right)+n^{1-\Omega(1)}$ bits of space, in the $w=\Theta(\lg n)$ word-RAM model.

. The main ingredient of both Theorem 1 and 2 is a new succinct data structure for computing RANK queries over the compressed BWT string $L=\operatorname{BWT}(x)$, with exponentially small redundancy $r \approx n / 2^{t}$ with respect to $|\operatorname{RLX}(L)|$ (see Theorem 3.1 below). Our data structure builds upon and is inspired by the work of Pătraşcu[32], who showed a similar exponential trade-off for the RANK problem, with respect to the zeroth order entropy $H_{0}(L)$, i.e., in the contextfree setting. In that sense, our work can be viewed as a certain higher-order entropy analogue of [32].

The most challenging part of our data structure is dealing with the Move-to-Front (MTF) encoding of $L$. This adaptive coding method comes at a substantial price: decoding the $i$ th character from its encoding $\operatorname{MTF}(x)_{i}$ requires the decoder to know the current "state" $S_{i} \in \mathcal{S}_{|\Sigma|}$ of the encoder, namely, the precise order of recently occurring symbols, which itself depends on the entire history $x_{<i}$. This feature of the MTF transform, that the codebook itself is dynamic, is a qualitative difference from other compression schemes such as Huffman coding or (non-adaptive) arithmetic codes, in which the codebook is fixed. In fact, in that sense the MTF transform is conceptually closer to the BWT transform itself than to Huffman or arithmetic codes, since in both transforms, decoding the $i$ th character is a sequential function of the decoded values of previous characters. Fortunately, it turns out that MTF has a certain local property that can be leveraged during preprocessing time, leading to the following key result:

Theorem 4 (Locally-decodable MTF, Informal). For any string $x \in \Sigma^{n}$, there is a succinct data structure that encodes $x$ using at most $H_{0}(\operatorname{MTF}(x))+\frac{n}{(\lg (n) / t)^{t}}+n^{0.9}$ bits of space, such that $x_{i}$ can be decoded in time $O(t)$. Moreover, it supports RANK queries with the same parameters.

The additive $n^{0.9}$ term stems from the storage space of certain look-up tables, which are shared across $n / r$ blocks of size $r=$ $(\lg (n) / t)^{t}$. So these tables occupy o(1) bits per block in an amortized sense. Theorem 4 therefore has the surprising corollary that, with only $O(1)$ bits of redundancy per block (even with respect to the MTF code followed by arithmetic coding), decoding time can be reduced from $\Omega(n)$ to $O(\lg n)$.

Techniques. Our techniques are quite different from those used in previous compressed text indexing literature, and in particular from the work of [13]. At a high-level, our main result uses a combination of succinct Predecessor search (under some appropriate encoding) along with ad-hoc design of two "labeled" B-ary tree data structures, known as augmented B-trees (see Section 2.4). We use a theorem of Pătraşcu[32] to compress these aB-trees, resulting in succinct representations while preserving query time. Our main tree structure relies on a new local preprocessing step of the MTF codeword, which in turn facilitates a binary-search algorithm for the dynamic MTF codebook (stack state). We show that this algorithm can be 'embedded' as a (compressed) augmented B-tree. Our cell-probe lower bound (Theorem 3.2) relies on a new "entropy polarization" lemma for BWT permutations, combined with a 'nonuniform' adaptation of Golynski's cell-elimination technique for the succinct permutations problem [17].

\subsection{Related Work}

A large body of work has been devoted to succinct data structures efficiently supporting RANK/SELECT and (easier) DictionARY queries under zeroth-order empirical entropy, i.e., using $H_{0}(x)+o(n)$ bits of space. In this "context-free" regime, early results showed a near-linear $r=\tilde{O}(n / t)$ trade-off between time and redundancy (e.g., $[5,18,31])$, and this was shown by Miltersen [27] to be optimal for systematic ${ }^{7}$ data structures. This line of work culminated with a surprising result of Pătraşcu[32], who showed that an exponential trade-off between time and redundancy can be achieved using non-systematic data structures in the word-RAM model, supporting all the aforementioned operations in query time $O(t)$ and $s \approx H_{0}(x)+O\left(n /(\lg (n) / t)^{t}\right)$ bits of space. For RANK/SELEct, this trade-off was shown to be optimal in the cell-probe model with word-size $w=\Theta(\lg n)$ [34], while for Dictionary (and MemberSHIP) queries, the problem is still open [7, 39, 40]. There are known string-matching data structures, based on context-free grammar compression (e.g., LZ or SLPs [4]), that achieve logarithmic query time for Dictionary queries, at the price of linear (but not succinct) space in the compressed codeword [4, 8]. However, these data structures have an $O(n / \lg n)$ additive space term, regardless of the compressed codeword, which becomes dominant in the $o(1)$ per-bit entropy regime, the interesting setting for this paper (see [10] for elaboration).

The problem of compressed pattern matching has been an active field of research for over four decades, since the works of McCreight [26] and Manber and Myers [23], who introduced suffix trees and suffix arrays. Ferragina and Manzini [13] were the first to achieve a compressed text index (with respect to higher-order entropy $H_{k}$ ), supporting pattern-matching counting and reporting queries in sublinear $(o(n))$ space and essentially optimal query time. Their BWT-based data structure, known as the FM-index, is still widely used in both theory and practice, and its applications in genomics go well beyond the scope of this paper [37]. Subsequent works, e.g. $[10,19,35]$, designed compressed text indices under other entropycoding space benchmarks, such as Lempel-Ziv compression, but to the best of our knowledge, all of them again require $\Omega(n / \lg n)$ bits of space, even when the text itself compresses to $o(n / \lg n)$ bits. We remark that for systematic data structures, linear trade-off $(r=\tilde{\Theta}(n / t))$ is the best possible for counting pattern-matches [16, 17], hence Theorem 2 provides an exponential separation between systematic and non-systematic data structures for this problem. Gagie et. al[15] exhibited a data structure requiring $O(\kappa \lg (n / \kappa))$ words of space ( $\kappa$ being the number of runs in BWT $(x)$ ), that reports the occ occurrences of a pattern in optimal time $O(n+o c c)$. For a more complete state of affairs on compressed text indexing, we refer the reader to $[13,30]$.

\footnotetext{
${ }^{7}$ Systematic data structures are forced to store the raw input database $x$, followed by an $r$-bit additional index.
} 
Another related problem to our work is the succinct permutations problem [17, 29, 41, 42], where the goal is to succinctly represent a permutation $\pi \in S_{n}$ using $\lg n !+r$ bits of space, supporting evaluation $(\pi(i))$ and possibly inverse $\left(\pi^{-1}(i)\right)$ queries in time $t$ and $q$ respectively. For the latter problem, an essentially tight trade-off $r=\Theta(n \lg n / t q)$ is known in the regime $t, q \in \tilde{\Theta}(\lg n)[17,29]$.

Organization. We start with some necessary background and preliminaries in Section 2. Section 3 provides a high-level technical overview of our main results. Sections 4,5 contain our main data structure and proofs of Theorems 1,2 and 4. We defer the proofs of the application to improved pattern-matching (reporting) queries (Theorem 3) and the cell-probe lower bound (Theorem 3.2) to the full version of our paper[38].

\section{BACKGROUND AND PRELIMINARIES}

For an $n$-letter string $x \in \Sigma^{n}$, let $n_{c}$ be the number of occurrences, i.e., the frequency, of the symbol $c \in \sum$ in $x$. For $1 \leq i<j \leq n$, let $x[i: j]$ denote the substring $\left(x_{i}, x_{i+1}, \cdots, x_{j}\right)$. For convenience, we use $x_{<i}$ to denote the prefix $\left(x_{1}, x_{2}, \cdots, x_{i-1}\right)$. The $k^{t h}$ context of a character $x_{i}$ in $x$ is the substring of length $k$ that precedes it. A run in a string $x$ is a maximal substring of repetitions of the same symbol. For a compression algorithm $\mathcal{A}$, we denote by $|\mathcal{A}(x)|$ the output size in bits. The zeroth order empirical entropy of the string $x$ is $H_{0}(x):=\sum_{c \in \Sigma} n_{c} \lg \frac{n}{n_{c}}$ (all logarithms throughout the paper are base-2, where by standard convention, $0 \lg 0=0$ ). We have $0 \leq H_{0}(x) \leq n \lg |\Sigma|$. For a substring $y \in \Sigma^{k}$, let $y_{x}$ denote the concatenated string consisting of the single characters following all occurrences of $y$ in $x$. The $k^{t h}$ order empirical entropy of $x$ is defined as $H_{k}(x):=\sum_{y \in \Sigma^{k}} H_{0}\left(y_{x}\right)$. This prior-free measure intuitively captures "conditional" entropies of characters in correlated strings with bounded context, and is a lower bound on the compression size $|\mathcal{A}(x)|$ of any $k$-local compressor $\mathcal{A}$; for elaboration see [24]. For all $k \geq 0$, we have $H_{k+1}(x) \leq H_{k}(x)$. Note that the space benchmark $H_{k}$ can be significantly smaller than $H_{0}$. For example, for $x=(a b)^{n / 2}, H_{0}(x)=n$ but $H_{k}(x)=0$ for any $k \geq 1$ (assuming the length $n$ is known in advance, and $\lg n$ otherwise). For a random variable $X \sim \mu, H(X)$ denotes the Shannon entropy of $X$. Throughout the paper, we assume the original alphabet size is $|\Sigma|=O(1)$.

Succinct data structures. We work in the word-RAM model of word-length $w=\Theta(\lg n)$, in which arithmetic and shift operations on memory words require $O(1)$ time. A succinct data structure for an input $x \in \Sigma^{n}$ is a data structure that stores a small additive space overhead $r=o(n)$ beyond the "information-theoretic minimum" space $h(x)$ required to represent $x$, while supporting queries efficiently. In the "prior-free" setting, $h(x)$ is usually defined in terms of empirical entropy $H_{k}(x)$. The space overhead $r$ is called the redundancy, and is measured in bits.

\subsection{The Burrows-Wheeler Transform}

Given a string $x \in \Sigma^{n}$, the Burrows-Wheeler Transform of $x$, denoted BWT $(x)$, is defined by the following process. We append a unique end-of-string symbol ' $\$$ ', which is lexicographically smaller than any character in $\Sigma$, to $x$ to get $x \$$ (without this technicality, invertibility is only up to cyclic shifts). We place all $n+1$ cyclic shifts of the string $x \$$ as the rows of an $(n+1) \times(n+1)$ matrix, denoted by $\widehat{\mathcal{M}}$. Then we sort the rows of $\widehat{\mathcal{M}}$ in lexicographic order. The sorted matrix, denoted $\mathcal{M}$, is henceforth called the "BWT matrix" of $x$. Finally, we output $L \in(\Sigma \cup\{\$\})^{n+1}$, the last column of the BWT matrix $\mathcal{M}$. We henceforth use the shorthand $L:=\mathrm{BWT}(x)$.

We observe that every column in $\mathcal{M}$ is a permutation of $x \$$. Let $F$ and $L$ be the first and last column of $\mathcal{M}$ respectively. See an example in Figure 1 below. For ease of notation, we shall refer to $\mathrm{x} \$$ as $x$, denote its length by $n$, and include $\$$ in $\Sigma$.
mississippi\$
ississippi\$m
ssissippi\$mi
sissippi\$mis
issippi\$miss
ssippi\$missi
sippi\$missis
ippi\$mississ
ppi\$mississi
pi\$mississip
i\$mississipp
\$mississippi

\begin{tabular}{ccc} 
F & & $\mathrm{L}$ \\
\cline { 2 - 3 } $\mathbf{\$}$ & mississipp & $\mathrm{i}$ \\
$\mathrm{i}$ & $\mathbf{\text { \$mississip }}$ & $\mathrm{p}$ \\
$\mathrm{i}$ & ppi\$missis & $\mathrm{s}$ \\
$\mathrm{i}$ & ssippi\$mis & $\mathrm{s}$ \\
$\mathrm{i}$ & ssissippi\$ & $\mathrm{m}$ \\
$\mathrm{m}$ & ississippi & $\mathbf{\$}$ \\
$\mathrm{p}$ & i\$mississi & $\mathrm{p}$ \\
$\mathrm{p}$ & pi\$mississ & $\mathrm{i}$ \\
$\mathrm{s}$ & ippi\$missi & $\mathrm{s}$ \\
$\mathrm{s}$ & issippi\$mi & $\mathrm{s}$ \\
$\mathrm{s}$ & sippi\$miss & $\mathrm{i}$ \\
$\mathrm{s}$ & sissippi\$m & $\mathrm{i}$
\end{tabular}

Figure 1: Burrows-Wheeler Transform for the string $x=$ "mississippi", with the unsorted matrix $\widehat{M}$ on the left and the sorted matrix $\mathcal{M}$ on the right. The output is $L=$ $\operatorname{BWT}(x)=$ "ipssm\$pissii”.

2.1.1 Decoding BWT and the "LF Mapping". While not obvious at first glance, BWT is an invertible transformation. An important first observation for this fact is that the first column $F$ of the BWT matrix $\mathcal{M}$ is actually known "for free" (as long as the frequencies of each symbol are stored, using negligible $O(|\Sigma| \lg n)$ additive space), since $\mathcal{M}$ is sorted lexicographically (See Figure 1 ). To see why this is useful, we first introduce the following central definition:

Definition 1 (Rank of a character). Let $y \in \Sigma^{n}$. For any $c \in \Sigma, i \in$ $[n], r k_{y}(c, i)$ denotes the number of occurrences of the symbol $c$ in the $i^{\text {th }}$ prefix $y_{\leq i}=y[1: i]$.

Note that $r k_{L}(c, n)=n_{c}$, recalling that $n_{c}$ is the frequency of $c$ in $x$. We define the Last-to-First (LF) column mapping $\pi_{L F}:[n] \mapsto[n]$ by setting $\pi_{L F}(i)=j$ if the character $L_{i}$ is located at $F_{j}$, i.e., $L_{i}$ is the first character in the $j^{t h}$ row of the BWT matrix $\mathcal{M}$. We note that $\pi_{L F}$ is a permutation.

An indispensable feature of BWT, the LF Mapping Property, states that for any character $c \in \sum$, the occurrences of $c$ in the first column $F$ and last column $L$ follow the same order. In other words, the permutation $\pi_{L F}$ preserves the order among all occurrences of $c$.

Fact 1 (LF Property). Fix an index $i \in[n]$, and let $c=L_{i}$. Then $r k_{L}(c, i)=r k_{F}\left(c, \pi_{L F}(i)\right)=\pi_{L F}(i)-\sum_{c^{\prime}<c} n_{c^{\prime}}$.

The second equality follows directly from the fact that the first column $F$ is sorted lexicographically by construction, while the first equality also requires the fact that $L$ is sorted by its right context. The formal argument is deferred to the full version[38]. The LF 
Mapping Property leads to the following lemma, which is the heart of the BWT decoding algorithm:

Lemma 1. Fix a data structure $D$ that returns $r k_{L}(c, i)$ for given $i \in[n], c \in \Sigma$. Let $j \in[n]$. Given the position $i$ of $x_{j}$ in $L$, then we can compute (even without knowing $j$ ) the character $x_{j}=L_{i}$, and (if $j \geq 2$ ) the position $i^{\prime}$ of $x_{j-1}$ in $L$, with $O(|\Sigma|)$ calls to $D$.

Proof. Given the position $i \in L$ of $x_{j}$, the character $L_{i}=x_{j}$ can be decoded via $2|\Sigma|$ rank queries on $L$, by computing $r k_{L}(c, i)-$ $r k_{L}(c, i-1) \forall c \in \Sigma$, which is nonzero only for $c^{*}:=x_{j}$. Now, given the rank $r k_{L}\left(c^{*}, i\right)$ of $x_{j}$ in $L$, the LF-property (Fact 1) allows us to translate it to the index $i^{\prime}:=\pi_{L F}(i)$ of $x_{j}$ in F. As such, $F_{i^{\prime}}=x_{j}$. But this means that $L_{i^{\prime}}=x_{j-1}$, as each row of $\mathcal{M}$ is a cyclic shift of $x$ (in each row, $L$ and $F$ contain consecutive characters of $x$ ).

The decoding argument asserts that a RANK data structure over $L$ allows us to "move back" one character in $x$. Note that the decoding algorithm implied by Lemma 1 is inherently sequential: decoding a single character $x_{n-i}$ of $x$ requires $O(|\Sigma| \cdot i)$ calls to $D$, hence $\Omega(n)$ worst-case time.

\subsection{Compressing BWT}

2.2.1 Move-to-Front encoding (MTF). As mentioned in the introduction, when reasonably short contexts tend to predict a character in the input text $x$, the BWT string $L=\mathrm{BWT}(x)$ will exhibit local similarity, i.e, identical symbols will tend to recur at close vicinity. As such, we expect the integer string $\operatorname{MTF}(L)$ to contain many small integers. This motivates the following relative encoding of $L$ :

The Move-to-Front transform (Bentley et al. 1986 [3]) replaces each character of $L$ with the number of distinct characters seen since its previous occurrence. Formally, the encoder maintains a list, called the MTF-stack, initialized with all characters $c \in \Sigma$ ordered alphabetically. To encode the $i^{t h}$ character, the encoder outputs its RANK in the current stack $S_{i-1} \in \mathcal{S}_{|\Sigma|}$ (with the character at the top of $S_{i-1}$ having RANK 0), and moves $c=L_{i}$ to the top of the stack, generating $S_{i}$. At any instant, the MTF-stack contains the characters ordered by recency of occurrence. Denote the output of this sequential algorithm by $m(L):=\operatorname{MTF}(L)=\left(m_{1}, m_{2}, \ldots, m_{n}\right) \in$ $\{0,1, \cdots,|\Sigma|-1\}^{n}$.

A few remarks are in order: First, note that runs of identical characters in $L$ are transformed into runs of 0 s (except the first character in the run) in the resulting string $m(L)$. Second, at each position $i \in[n]$, the corresponding MTF-stack $S_{i}$ defines a unique permutation $\pi_{i} \in \mathcal{S}_{|\Sigma|}$ on $[|\Sigma|]$.

\subsection{The RLX compression benchmark}

Based on the MTF transform and following the original paper of Burrows and Wheeler [6], [13, 24] analyzed the following compression algorithm ${ }^{8}$ to encode $L=\operatorname{BWT}(x)$, henceforth denoted $\operatorname{RLX}(L)$ :

(1) Apply the Move-to-Front transform on $L$ to get $\operatorname{MTF}(L)$.

(2) Denote by $L^{\text {runs }}$ the concatenation of substrings of $\operatorname{MTF}(L)$ corresponding to runs of $0 s$, and by $\operatorname{MTF}\left(L^{- \text {runs }}\right):=[n] \backslash$ $L^{\text {runs }}$ the remaining indices. Encode all 0-runs in $L^{\text {runs }}$ using Run-Length encoding, where each run is replaced by its

\footnotetext{
${ }^{8}$ Excluding the final arithmetic coding step.
}

$$
\begin{aligned}
L & =\text { "i c a a a t h e e e e e e t h e u u". } \\
\operatorname{MTF}(L) & =(8,3,2, \mathbf{0}, \mathbf{0}, 19,9,7, \mathbf{0}, \mathbf{0}, \mathbf{0}, \mathbf{0}, \mathbf{0}, 2,2,2,20, \mathbf{0}) . \\
\operatorname{RLX}(L) & =(8,3,2,2,19,9,7, \mathbf{5}, 2,2,2,20, \mathbf{1})
\end{aligned}
$$

Figure 2: Illustration of MTF and RLE encoding, with RLE symbols in $\operatorname{RLX}(L)$ in bold font. The final prefix-free code is not shown. MTF exploits local context in $L$, leading to small integers and in particular, 0-runs. Each 0-run is encoded by its length (a single character) in $\operatorname{RLX}(L)$, yielding a significantly shorter string.

length (encoded using a prefix-free code), and denote the output by RLE ( $\left.L^{\text {runs }}\right)$.

(3) Encode the remaining (non-runs) symbols in $\operatorname{MTF}\left(L^{-r u n s}\right)$ using a 0-order entropy $\operatorname{code}^{9}$ (e.g., Huffman or Arithmetic coding), to obtain the final bit stream RLX $(L)$ (modified to be prefix-free over the alphabet comprising non-zero MTF symbols and run-length symbols).

See illustration in Figure 2. By a slight abuse of notation, the output length of the algorithm ${ }^{10}$ is

$$
|\operatorname{RLX}(L)|=\left|\operatorname{RLE}\left(L^{\text {runs }}\right)\right|+\left\lceil H_{0}\left(\operatorname{MTF}\left(L^{-r u n s}\right)\right)\right\rceil .
$$

\subsection{Augmented B-Trees [32]}

Central to our data structure is the notion of "augmented B-trees", or $a B$-trees for short. Let $B \geq 2, t \in \mathbb{N}$, and let $A \in \Sigma^{S}$ be an array of length $s:=B^{t}$. An aB-tree $\mathcal{T}$ over $A$ is a $B$-ary tree of height $t$, with leaves corresponding to elements of $A$. Each node $v \in \mathcal{T}$ is augmented with a value $\varphi_{v}$ from an alphabet $\Phi$. This value $\varphi_{v}$ must be a function of the subarray of $A$ corresponding to the leaves of the subtree $\mathcal{T}_{v}$ rooted at $v$. In particular, the value of a leaf must be a function of its array element, and the value of an internal node must be a function of the values of its $B$ children.

The query algorithm starts at the root and traverses down the tree along a potentially adaptive path. When it visits a node, it reads all the values of its $B$ children and recurses to one of them, until it reaches a leaf node and returns the answer. We ensure the query algorithm spends $O(1)$ time per node, by packing all augmented values of the children in one word.

For a given aB-tree $\mathcal{T}$ and value $\varphi \in \Phi$, let $\mathcal{N}(s, \varphi)$ be the number of possible arrays $A \in \Sigma^{s}$ such that the root is labeled with $\varphi$. A reasonable information-theoretic space benchmark for this data structure, conditioned on the root value $\varphi$, is therefore $\lg \mathcal{N}(s, \varphi)$. Pătraşcu proved the following remarkable result, which allows to compress any aB-tree, while preserving its query time:

Theorem 5 (Compressing aB-trees, [32]). Let $B=O\left(\frac{w}{\lg (s+|\Phi|)}\right)$. We can store an aB-tree of size $s$ with root value $\varphi$ using $\lg \mathcal{N}(s, \varphi)+2$ bits. The query time is $O\left(\lg _{B} s\right)=O(t)$, assuming precomputed lookup tables of $O\left(|\Sigma|+|\Phi|^{B+1}+B \cdot|\Phi|^{B}\right)$ words, which only depend on $s, B$ and the aB-tree query algorithm.

\footnotetext{
${ }^{9}$ A 0 -order encoder assigns a unique bit string to each symbol independent of its context, such that we can decode the concatenation of these bit strings.

${ }^{10}$ up to prefix-free coding overheads.
} 
The proof idea is to use recursion in order to encode the root value $\varphi_{r}$, followed by an encoding of the augmented values $\varphi_{v}$ of every child of the root, "conditioned" on $\varphi_{r}$, and so on, without losing (almost) any entropy (recursive encoding is needed to achieve this, since $\mathcal{N}\left(s, \varphi_{r}\right)$ may not be a power of 2). Theorem 5 allows us to represent any aB-tree with a redundancy of merely 2 bits (over the zeroth-order empirical entropy of the leaves). Since the extra look-up tables do not depend on the array $A$, in our application, we use a similar trick as in [32] and divide the original array of length $n$ into blocks of length $s=B^{t}$, building an aB-tree over each block. We then invoke Theorem 5 separately on each tree, adding an auxiliary data structure that aggregates query answers across blocks to answer the query on the original array (for further details, see [32]). Beyond facilitating the desired query time, this application renders the extra space occupied by the look-up tables in Theorem 5 inconsequential, as they can be shared across blocks. We remark that this "splitting" trick of [32] only applies when the augmented values $\varphi$ are composable, in the sense that $\varphi(A \circ B)=f(\varphi(A), \varphi(B))$, where $A \circ B$ is the concatenation of the arrays $A, B$. The aB-trees we design shall use augmented vector values which are (componentwise) composable.

\section{TECHNICAL OVERVIEW}

Both Theorem 1 and Theorem 2 follow from the next result, which is the centerpiece of this work:

Theorem 3.1. There exists a small constant $\delta>0$ such that for any $x \in \Sigma^{n}$ and $t^{\prime} \leq \delta \lg n$, there is a succinct data structure $\mathcal{D}_{r k}$ that supports RANK queries on $L=\mathrm{BWT}(x)$ in time $O\left(t^{\prime}\right)$, using at most $|\mathrm{RLX}(L)|+n \lg n / 2^{t^{\prime}}+n^{1-\Omega(1)}$ bits of space, in the $w=\Theta(\lg n)$ word-RAM model.

Theorem 2 is a direct corollary of Theorem 3.1, as one can count the number of occurrences of a given pattern $p \in \Sigma^{\ell}$ in $x$, using $O(\ell)$ successive RaNK queries on $L$ (see [13]).

To see how Theorem 1 follows from Theorem 3.1, consider the following data structure for locally-decoding an index $x_{i}$ of $x$ in time $t$ : Let $t^{\prime}<t$ be a parameter to be determined shortly. Let $\mathcal{D}_{r k}$ be the data structure supporting rank queries on $L$ in time $O\left(t^{\prime}\right)$. We divide $x$ into $\lceil n / T\rceil$ blocks of size $T:=O\left(t / t^{\prime}\right)$, and store, for each ending index $j$ of a block, the position in $L$ corresponding to $x_{j}$. In other words, we simply record "shortcuts" of the BWT transform after every block of size $T$. Given an index $i \in[n]$, the data structure computes the endpoint $j:=\left\lceil\frac{i}{T}\right\rceil T$ of the block containing $i$, reads from memory the position of $x_{j}$ in $L$, and simulates $(j-i) \leq T=$ $O\left(t / t^{\prime}\right)$ sequential steps of the LF-mapping decoding algorithm from Section 2.1.1, to decode $x_{i}$. By Lemma 1, each step requires $O(|\Sigma|)$ Rank queries on $L$, each of which can be done using $\mathcal{D}_{r k}$ in $O\left(t^{\prime}\right)$ time, so the overall running time is $O\left(T \cdot t^{\prime}\right)=O(t)$. To balance the redundancy terms, observe that the overall space of our data structure (up to $O\left(n^{\varepsilon}\right)$ terms) is

$$
s=|\operatorname{RLX}(L)|+\frac{n \lg n}{2^{t^{\prime}}}+\frac{n \lg n}{T} .
$$

Thus, setting $t^{\prime}=\Theta(\lg t)$, leads to overall redundancy $r=O\left(\frac{n \lg n \lg t}{t}\right)=\tilde{O}\left(\frac{n \lg t}{t}\right)$, as claimed in Theorem 1. Next, we provide a high-level overview of the proof of Theorem 3.1.

\subsection{Proof Overview of Theorem 3.1}

Recall (Section 2.2), that the RLX compression of $L=\mathrm{BWT}(x)$ can be summarized as :

$$
|\operatorname{RLX}(L)|=\left|\operatorname{RLE}\left(L^{\text {runs }}\right)\right|+\left\lceil H_{0}\left(\operatorname{MTF}\left(L^{-r u n s}\right)\right)\right\rceil .
$$

Since RLX compresses the two parts $L^{\text {runs }}$ and $L^{-r u n s}$ using two conceptually different encodings (RLE and MTF, respectively), it makes sense to design a RANK data structure for each part separately (along with an efficient mapping for combining the two answers to compute the overall rank of a character in $L$ ). This modular approach simplifies the presentation and, more importantly, enables us to achieve a significantly better redundancy for Theorem 4 (i.e., $n /(\lg n / t)^{t}$ instead of $\left.n / 2^{t}\right)$, but is slightly suboptimal in terms of space (by an $\Omega(|\operatorname{RLX}(L)|$ ) additive term). In the actual proof, we show how the two data structures below can be "merged" to avoid this overhead.

A Rank data structure over $\operatorname{RLE}\left(L^{\text {runs }}\right)$. Our first goal is to design a compressed data structure $\mathcal{D}_{\mathrm{RLE}}$ that reports, for each symbol $c \in \sum$ and index $i \in[n]$, the number of occurrences of $c$ in $L[1: i]$ that are contained in $L^{\text {runs }}$, i.e., the number of consecutive 0's in $\operatorname{MTF}(L)[1: i]$ corresponding to runs of $c$. As RLX represents this substring by "contracting" each run into a singleton (denoting its length), solving this problem succinctly essentially entails a Predecessor search ${ }^{11}$ on the universe $[n]$ with $\kappa=\kappa(L)$ "keys", where $\kappa$ denotes the number of runs in $L$. Alas, under the standard representation of this input, as a $\kappa$-sparse string in $\{0,1\}^{n}$, Predecessor search clearly requires at least $\lg \left(\begin{array}{l}n \\ \kappa\end{array}\right)$ bits of space [32, 33], which could be $\gg|\operatorname{RLX}(L)|$ (for example, when all but a single 0-run are of constant length and separation, which is an oblivious feature to the previous representation). To adhere to the RLE space benchmark, we use a more suitable alternative representation of $L^{\text {runs }}$.

To this end, suppose for simplicity of exposition, that $L$ consists entirely of runs (i.e., $L=L^{r u n s}$ ), and that the character $c \in \Sigma$ corresponding to each 0-run is known at query time (this will be handled in the integrated data structure in Section 5). For $i \in$ $[\kappa]$, let $\ell_{i} \in[n]$ denote the length of the $i^{t h}$ run, and let $L^{\prime}=$ $\left(\ell_{1}, \ell_{2}, \ldots, \ell_{\kappa}\right) \in[n]^{\kappa}$ be the string that encodes the run lengths. Note that RLX spends precisely $\sum_{i} \lg \ell_{i}$ bits to encode this part (ignoring prefix-coding issues).

To compute $r k_{L^{\text {runs }}}(c, i)$, we design an adaptive augmented Btree, that essentially implements a predecessor search over the new representation $L^{\prime}$ of $L^{\text {runs }}$ : We first construct a $B$-tree $\mathcal{T}$ over the array $L^{\prime} \in[n]^{\kappa}$, and augment each intermediate node $v$ of the tree with the (vector-valued) function $\varphi_{R L E}(v):=\left(\varphi_{\ell}^{c}(v)\right)_{c \in \Sigma} \in[n]^{|\Sigma|}$ , where $\varphi_{\ell}^{c}(v)$ counts the total sum $\sum_{j \in \mathcal{T}_{v}} \ell_{j}$ of run-lengths in the subtree of $v$, corresponding to runs of $c$. Given an index $i \in[n]$ and character $c \in \Sigma$, the query algorithm iteratively examines the labels of all $B$ children of a node $v \in \mathcal{T}$ starting from the root, and recurses to the rightmost child $u$ of $v$ for which $\sum_{c} \varphi_{\ell}^{c}(u) \leq i$ (i.e., to the subtree that contains the interval $\ell_{j}$ to which $i$ belongs), collecting the sum of $\varphi_{\ell}^{c}(u)$ 's along the query path.

To ensure query time $O\left(t^{\prime}\right)$, we break up the array as in [32] into sub-arrays each of size $B^{t^{\prime}}$ (for $B=\Theta(1)$ ), and build the aforementioned tree over each sub-array (this is possible since the augmented

${ }^{11}$ For a set of keys $S \subset \mathcal{U}$ with $|S|=\kappa, \operatorname{Predecessor}(i, S)$ returns $\max \{x \in S \mid x \leq$ $i\}$. 
vector $\varphi_{R L E}$ is a (component-wise) composable function). To ensure the desired space bound for representing $\mathcal{T}$, we further augment each node $v$ with a "zeroth-order entropy" constraint $\varphi_{0}(v)$, counting the sum of marginal empirical entropies $n_{c}^{v} \lg \left(n_{c} / n\right)^{12}$ of the elements in the subtree $\mathcal{T}_{v}$ (which can be done recursively due to additivity of $\varphi_{0}$ w.r.t $v$ 's). A standard packing argument then ensures $\mathcal{N}(\kappa, \varphi) \leq 2^{\varphi_{0}(r)} \lesssim 2^{H_{0}\left(\mathcal{T}_{r}\right)}$ (where $\mathcal{T}_{r}$ is the sub-array rooted at $r$ ), as desired. We then invoke Theorem 5 to compress $\mathcal{T}$ to $H_{0}\left(L^{\prime}\right)+O\left(\frac{n \lg n}{B^{t^{\prime}}}\right)$ bits, yielding exponentially small redundancy (up to $n^{1-\varepsilon}$ additive terms). This ensures that the total space (in bits) occupied by $\mathcal{D}_{\mathrm{RLE}}$ is essentially

$$
H_{0}\left(L^{\prime}\right)+O\left(\frac{n \lg n}{B^{t^{\prime}}}\right) \leq\left|\operatorname{RLE}\left(L^{\text {runs }}\right)\right|+O\left(\frac{n \lg n}{B^{t^{\prime}}}\right) .
$$

The actual proof is slightly more involved, since the merged data structure needs to handle characters from both $L^{\text {runs }}$ and $\operatorname{MTF}\left(L^{-r u n s}\right)$ simultaneously, hence it must efficiently distinguish between 0-runs corresponding to different symbols. Another issue is that Theorem 5 of [32] is only useful for truly sub-linear alphabet sizes, whereas $\left(L^{\prime}\right)_{i} \in[n]$, hence in the proof we must also split long runs into chunks of length $\leq n^{\varepsilon}$. A simple application of the $\log$-sum inequality ensures this truncation increases space by only an $\tilde{O}\left(n^{1-\varepsilon}\right)$ additive term.

A Rank data structure over $\operatorname{MTF}\left(L^{-r u n s}\right)$. The more challenging task is computing $r k_{L^{-r u n s}}(c, i)$, i.e., the frequency of $c$ in $L[1$ : $i$ ] contained in the substring $\operatorname{MTF}\left(L^{-r u n s}\right)$, which is obtained by applying the MTF transform to $L$ and deleting all 0-runs (see Figure 2). Note that the mapping from $i \in L$ to its corresponding index $i^{\prime} \in \operatorname{MTF}\left(L^{-r u n s}\right)$ amounts to subtracting all runs before $i$. This operation can be performed using a single partial-sum query to our integrated data structure (in Section 5), which collects the sum of $\varphi_{\ell}^{c}(u)$ 's over all $c \in \Sigma$ along the query path.

As discussed in the introduction, the adaptive nature of the MTF encoding has the major drawback that decoding the $j^{\text {th }}$ symbol $\operatorname{MTF}\left(L^{-r u n s}\right)_{j}$, let alone computing its rank, requires knowing the corresponding MTF stack state $S_{j-1} \in \mathcal{S}_{|\Sigma|}$ (i.e., the precise order of recently occurring symbols), which itself depends on the entire history $L_{<j}^{-r u n s}$. A straightforward solution is to store the MTF stack state after every block of length $t^{\prime}$ (where $t^{\prime}$ is the desired query time), much like the "marking" solution for decoding BWT, yielding a linear search for the stack-state $S_{j}$ from the nearest block, and thus a linear time-space trade-off.

To speed up the search for the local stack-state, we observe the following key property of the MTF transform: Let $\operatorname{MTF}(x):=$ $\left(m_{1}, m_{2}, \ldots, m_{n}\right)$ be the MTF transform of $x \in \Sigma^{n}$ (see Figure 2 for illustration). Let $\mathcal{I}=[i, j]$ be any sub-interval of $[n]$, and denote by $S_{i-1}, S_{j} \in \mathcal{S}_{|\Sigma|}$ the corresponding stack-states at the start and endpoints of $\mathcal{I}$. Now, consider the permutation $\pi_{\mathcal{I}}:=\mathbf{I d}_{\Sigma} \mapsto \widehat{S_{j}}$, obtained by simulating the MTF decoder on $\left(m_{i}, \ldots, m_{j}\right)$ starting from the identity state $\mathbf{I d}_{\Sigma}$, i.e., "restarting" the MTF decoding algorithm but running it on the encoded substring $\left(\operatorname{MTF}(x)_{i}, \ldots, \operatorname{MTF}(x)_{j}\right)$, arriving at some final state $\left(\widehat{S_{j}}\right)$ at the end of $\mathcal{I}$ (note that this process is well-defined). Then the true stack-state $S_{j}$ satisfies: $S_{j}=\pi_{I} \circ S_{i-1}$.

\footnotetext{
${ }^{12}$ For $c \in \sum$ and node $v, n_{c}^{v}$ denotes the frequency of $c$ in the sub-array rooted at $v$
}

The crucial point is that $\pi_{\mathcal{I}}$ is independent of the (true) stack state $S_{i-1}$, i.e., it is a local function of $\operatorname{MTF}(x)_{\mathcal{I}}$ only.

We show that this "decomposition" property of the MTF transform (Proposition 1), facilitates a binary search for the local stackstate $S_{j-1}$ (rather than linear search) with very little space overhead, as follows: At preprocessing time, we build an augmented $B$-tree over the array $\operatorname{MTF}\left(x_{1}, \ldots, x_{n}\right)$, where each intermediate node $v$ is augmented with the permutation $\pi_{v} \in \mathcal{S}_{|\Sigma|}$ corresponding to its subtree $\operatorname{MTF}\left(\mathcal{I}_{v}\right)$, obtained by "restarting" the MTF decoder to the identity state $\mathbf{I d}_{\Sigma}$, and simulating the MTF decoder from start to end of $\mathcal{I}_{v}$, as described above. Note that this procedure is well defined, and that the aforementioned observation is crucially used here, as the definition of aB-trees requires each augmented value of an intermediate node to be a local function of its own subtree. At query time, the query algorithm traverses the root-to-leaf $(j)$ path, composing the corresponding (possibly inverse) permutations between the stack-states along the path, depending on whether it recurses to a right or left subtree. We show this process ensures that when the query algorithm reaches the leaf $\operatorname{MTF}(x)_{j}$, it knows the correct stack-state $S_{j-1}$, and hence can correctly decode $x_{j}$. While this algorithm supports only "local decoding" (Dictionary) queries, with an extra simple trick, the above property facilitates a similar aB-tree supporting RANK queries under the MTF encoding (see Section 4).

Once again, in order to impose the desired space bound $(\approx$ $\left.H_{0}\left(\operatorname{MTF}\left(L^{- \text {runs }}\right)\right)\right)$ and to enable arbitrary query time $t^{\prime}$, we augment the nodes of the tree with an additional zeroth-order entropy constraint, and break up the array into sub-arrays of size $\Theta\left(B^{t^{\prime}}\right)$, this time for $B \approx \frac{\lg n}{t^{\prime}}$. Compressing each tree using Theorem 5 , and adding an auxiliary data structure to aggregate query answers across sub-arrays, completes this part and establishes Theorem 4.

\subsection{Lower Bound Overview}

We prove the following cell-probe lower bound for a somewhat stronger version of Problem 1, which requires the data structure to efficiently decode both forward and inverse dispositions of the induced BWT permutation between $X$ and $L:=\mathrm{BWT}(X)$ (we note that both the FM-Index and our data structure from Theorem 1 satisfy this natural requirement ${ }^{13}$, and elaborate on it in the full version [38]):

Theorem 3.2. Let $X \in_{R}\{0,1\}^{n}$ and let $\Pi_{X} \in \mathcal{S}_{n}$ be the induced $B W T$ permutation from indices in $L:=\operatorname{BWT}(X)$ to indices in $X$. Then, any data structure that computes $\Pi_{X}(i)$ and $\Pi_{X}^{-1}(j)$ for every $i, j \in[n]$ in time $t, q$ respectively, such that $t \cdot q \leq \delta \lg n / \lg \lg n$ (for some constant $\delta>0$ ), in the cell-probe model with word size $w=\Theta(\lg n)$, must use $n+\Omega(n / t q)$ bits of space in expectation.

We stress that Theorem 3.2 is more general, as our proof can yield nontrivial lower bounds against general (non-product) distributions $\mu$ on $\Sigma^{n}$ with "sufficient block-wise independence", though a lower bound against uniform strings is in some sense stronger, as it states that the above redundancy cannot be avoided even if $\Pi_{X}$ is stored in uncompressed form.

\footnotetext{
${ }^{13}$ I.e., for these data structures, we can achieve $q=O(t)$ by increasing the redundancy $r$ by a mere factor of 2 .
} 
Our proof of Theorem 3.2 is based on a "nonuniform" variation of the "cell-elimination" technique of [17], who used it to prove a lower bound of $r \geq \Omega(n \lg n / t q)$ on the space redundancy of any data structure for the succinct permutations problem PeRMs $_{n}$. In this problem, the goal is to represent a random permutation $\Pi \in_{R} \mathcal{S}_{n}$ succinctly using $\lg n !+o(n \lg n)$ bits of space, supporting forward and inverse evaluation queries in query times $t, q$ respectively, as above. Alas, [17]'s compression argument crucially requires that

$$
t, q \leq O\left(\frac{H(\Pi)}{n \cdot \lg \lg n}\right) .
$$

When $\Pi$ is a uniformly random permutation, i.e., $H(\Pi) \approx n \lg n$, this condition implies that the lower bound holds for $t, q \leq O(\lg n / \lg \lg n)$. In contrast, the BWT permutation of $X$ can have at most $n \lg |\Sigma|=$ $O(n)$ bits of entropy for constant-size alphabets (as $\Pi_{X}$ is determined by $X$ itself), hence condition (3) does not yield any lower bound whatsoever for our problem.

To overcome this obstacle, we prove an "entropy polarization" lemma for BWT: It turns out that for a random string $X$, while an average coordinate $\Pi_{X}(i)$ indeed carries only $O(1)$ bits of entropy, the entropy distribution has huge variance. In fact, we show that for any $\varepsilon \geq \tilde{\Omega}(1 / \lg n)$, there is a subset $\mathcal{I}$ of only $(1-\varepsilon) \frac{n}{\lg n}$ coordinates in $[n]$, whose total entropy is $H\left(\Pi_{X}(\mathcal{I})\right) \geq(1-O(\varepsilon)) n$, i.e., this small set of coordinates has maximal entropy $(\approx \lg n$ bits each), and essentially determines the entire BWT permutation ${ }^{14}$. This lemma is reminiscent of wringing lemmas in information theory [2], and may be a BWT property of independent interest in other applications.

The intuition behind the proof is simple: Consider dividing $X$ into $s:=\frac{n}{C \lg n}$ disjoint blocks of size $C \lg n$ each, and let $\mathcal{I}:=$ $\left\{I_{i}, \ldots, I_{s}\right\} \subset[n]$ denote the set of first coordinates in each block respectively. As $X$ is random, each of the $s$ blocks is an independent random $(C \lg n)$-bit string, so for a large enough constant $C$, with overwhelming probability these substrings will be distinct, and in particular, their lexicographic order will be uniquely determined. Conditioned on this likely event, this lexicographic ordering remains random, so the BWT locations of these indices alone must recover this random ordering, which is worth $\Omega(s \lg s)=\Omega(n)$ bits of information. However, the birthday paradox requires that $C>2$ to avoid collisions, in which case the above argument can only show that a small constant fraction $(<0.5 n)$ of the total entropy can be "extracted" from this small set, while the remaining $n-o(n)$ coordinates possess most of the entropy. Unfortunately, this guarantee is too weak for our "cell-elimination" argument, and would only yield a trivial $\Omega(n)$ space lower bound, while we are seeking a lower bound on the additive redundancy beyond $n$.

To bypass this, we observe that setting $C=(1+\varepsilon)$, the number of "colliding" blocks (i.e., non-distinct substrings of length $(1+\varepsilon) \lg n)$ is still only $\tilde{O}\left(n^{1-\varepsilon}\right) \ll \varepsilon n / \lg n$ with high probability. Moreover, we show that conditioned on this event $\mathcal{E}$, the lexicographic ordering among the remaining distinct $\approx(1-2 \varepsilon) \frac{n}{\lg n}$ blocks remains random. (For uniform $n$-bit strings, conditioning on $\mathcal{E}$ preserves exact uniformity of the ordering, by symmetry of $\mathcal{E}$ w.r.t block-permutation, but

\footnotetext{
${ }^{14}$ Note that here we view $\Pi_{X}$ as a mapping from $X$ to $L=\mathrm{BWT}(X)$ and not the other way around, but this is just for the sake of simplicity of exposition and looking at $\Pi_{X}^{-1}$ is of course equivalent.
}

more generally, we note that for any prior distribution, conditioning on $\mathcal{E}$ does not "distort" the original distribution by more than $\approx \sqrt{\lg (1 / \operatorname{Pr}[\mathcal{E}])}=o(1)$ in statistical distance, hence this argument can be generalized to nonuniform strings). Since, conditioned on $\mathcal{E}$, the BWT mapping on $\mathcal{I}$ determines the lexicographic ordering of the blocks, the data processing inequality (DPI) implies that the entropy of $\Pi_{X}(\mathcal{I})$ is at least $\approx(1-2 \varepsilon) \frac{n}{\lg n} \cdot \lg \frac{n}{\lg n} \geq(1-3 \varepsilon) n$, as claimed.

Applying the "entropy polarization" lemma with $\varepsilon=O(\lg \lg n / \lg n)$, we then show how to adapt [17]'s cell-elimination argument to nonuniform permutations, deleting 'unpopular' cells and replacing them with an efficient encoding of the partial bijection $\Pi_{X}(\mathcal{I})$ between (forward and inverse) queries $\in \mathcal{I}$ probing these cells. The polarization lemma then ensures that the remaining map of $\Pi_{X}$ on $\overline{\mathcal{I}}=[n] \backslash \mathcal{I}$ can be encoded directly using $H\left(\Pi_{X}(\overline{\mathcal{I}}) \mid \mathcal{I}, \Pi_{X}(\mathcal{I})\right) \leq$ $O(\varepsilon n)=O(n \lg \lg n / \lg n)$ bits, which will be dominated by the redundancy we obtain from the compression argument (as long as $t q \lesssim \lg n / \lg \lg n$ ), thereby completing the proof. Due to space constraints, we defer the detailed proof of Theorem 3.2 to the full version [38].

\section{RANK DATA STRUCTURE OVER MTF}

In this section, we prove the following theorem, which is a more formal version of Theorem 4 .

Theorem 6. For any string $x \in \Sigma^{n}$ with $|\Sigma|=O(1)$, there is a succinct data structure that encodes $x$ using at most

$$
H_{0}(\operatorname{MTF}(x))+n /\left(\frac{\lg n}{\max (t, \lg \lg n)}\right)^{t}+n^{1-\Omega(1)}
$$

bits of space, supporting RANK and DICTIONARY queries in time $O(t)$, in the word-RAM model with word size $w=\Theta(\lg n)$.

Setup and Notation. Let $\Sigma=\left\{c_{1}, c_{2}, \cdots, c_{|\Sigma|}\right\}$, where $c_{1}<c_{2}<$ $\cdots<c_{|\Sigma|}$ according to the lexicographical ordering on $\Sigma$. Let $S=$ $\left(a_{1}, a_{2}, \cdots, a_{|\Sigma|}\right)$ denote the MTF stack with $a_{1}$ at the top and $a_{|\Sigma|}$ at the bottom. For $j \in[|\Sigma|]$, let $S[j]$ denote the character at position $j$ in $S$, starting from the top. Fix a string $x=\left(x_{1}, x_{2}, \cdots, x_{n}\right) \in \Sigma^{n}$. Let $m=\operatorname{MTF}(x)=\left(m_{1}, m_{2}, \cdots, m_{n}\right) \in\{0,1, \cdots,|\Sigma|-1\}^{n}$ be the Move-to-Front (MTF) encoding of $x$, with the initial MTF stack $S_{0}:=\left(c_{1}, c_{2}, \cdots, c_{|\Sigma|}\right)$.

Given a MTF stack $S=\left(a_{1}, a_{2}, \cdots, a_{|\Sigma|}\right)$ and a permutation $\pi \in \mathcal{S}_{|\Sigma|}$, let $S^{\prime}=\pi \circ S$ be the stack such that $S^{\prime}[\pi(j)]=S[j]=a_{j}$ for all $j \in[|\Sigma|]$. We also associate with $S$ the permutation $\pi(S)$ which converts the initial stack $S_{0}$ to $S$, i.e., $S=\pi(S) \circ S_{0}$. In this sense, we say that $S_{0}$ corresponds to the identity permutation $\operatorname{Id}_{|\Sigma|}$ on $[|\Sigma|]$, as $S_{0}[j]=c_{j}$ for all $j \in[|\Sigma|]$. For $i \in[n]$, let $S_{i}$ be the stack induced by simulating the MTF decoder on $m[1: i]$, starting from $S_{0}$. Equivalently, $S_{i}$ is the stack induced by $\operatorname{MTF}(x[1: i])$, i.e., the stack just after encoding the first $i$ characters of $x$, starting from $S_{0}$. For $0 \leq i<j \leq n$, let $\pi_{i, j} \in \mathcal{S}_{|\Sigma|}$ be the unique permutation induced by simulating the MTF decoder on $m[i+1: j]$, starting from $S_{i}$.

\subsection{Properties of MTF Encoding}

The following proposition shows that for any $0 \leq i<j \leq n$, the permutation $\pi_{i, j}$ is a local function of $m[i+1: j]$. So, these 
permutations $\pi_{i, j}$ are valid augmented values for an aB-tree built over $m=\operatorname{MTF}(x)$, without reference to the MTF stacks $S_{i}$ and $S_{j}$.

Proposition 1. Fix $0 \leq i<j \leq n$, and let $S_{i}, S_{j}$ and $\pi_{i, j} \in \mathcal{S}_{|\Sigma|}$ be as defined above. Then $\pi_{i, j}$ is independent of $S_{i}$ and $S_{j}$, given $m[i+1: j]$. So, we can generate $\pi_{i, j}$ by simulating the MTF decoding algorithm on $m[i+1: j]$, starting from the identity stack $S_{0}$.

Proof. We prove this proposition by induction on $j-i$. Consider the base case, when $j-i=1$. Then by definition of a single MTF step, we have

$$
\pi_{i, i+1}(k)= \begin{cases}k+1 & \text { if } k \leq m_{i+1} \\ 1 & \text { if } k=m_{i+1}+1 \\ k & \text { if } k>m_{i+1}+1\end{cases}
$$

Clearly, $\pi_{i, i+1}$ is independent of $S_{i}$ and $S_{i+1}$ given $m_{i+1}$. This proves the base case. Now, suppose the claim is true for all $i, j$ such that $j-i=k \in \mathbb{N}$, and let $i, j$ be such that $j-i=k+1$. Then by the induction hypothesis, $\pi_{i, j-1}$ is independent of $S_{i}$ and $S_{j-1}$ given $m[i+1: j-1]$. Moreover, $\pi_{j-1, j}$ is independent of $S_{j-1}$ and $S_{j}$ given $m_{j}$. Due to the sequential nature of the MTF encoding, we clearly have

$$
\pi_{i, j}=\pi_{j-1, j} \circ \pi_{i, j-1}
$$

As both permutations $\pi_{j-1, j}$ and $\pi_{i, j-1}$ are independent of stacks $S_{i}, S_{j-1}, S_{j}$ given $m[i+1: j]$, the same is true for $\pi_{i, j}$.

The following expression captures the evolution of the MTF stack, for all $0 \leq i<j \leq n$ :

$$
S_{j}=\pi_{i, j} \circ S_{i}
$$

We can also "reverse" the steps of the MTF encoding. For $0 \leq$ $i<j \leq n$, given the final stack $S_{j}$ and the permutation $\pi_{i, j}$, we can recover the initial stack $S_{i}$ by inverting $\pi_{i, j}$ :

$$
S_{i}=\pi_{i, j}^{-1} \circ S_{j}
$$

\subsection{Locally Decodable MTF Code}

We first describe the construction of a single aB-tree $\mathcal{T}^{\prime}$ over the entire MTF encoding $m=\operatorname{MTF}(x) \in\{0,1, \cdots,|\Sigma|-1\}^{n}$, which supports "local decoding" (Dictionary) queries. Let $B \geq 2$ be the branching factor. Each node $v$ will be augmented with a permutation $\varphi_{\pi}(v) \in \Phi_{\pi}=\mathcal{S}_{|\Sigma|}$. For $i \in[n]$, the leaf node $v$ corresponding to $m_{i}$ is augmented with the permutation $\varphi_{\pi}(v)=\pi_{i-1, i}$. An internal node $v$ with children $v_{1}, v_{2}, \cdots, v_{B}$ (in order from left to right) is augmented with the composition of permutations of its children:

$$
\varphi_{\pi}(v)=\varphi_{\pi}\left(v_{B}\right) \circ \varphi_{\pi}\left(v_{B-1}\right) \circ \cdots \circ \varphi_{\pi}\left(v_{1}\right) .
$$

We observe that a node $v$ whose subtree $\mathcal{T}_{v}^{\prime}$ is built over the subarray $m[i+1: j]$ is augmented with the value $\varphi_{\pi}(v)=\pi_{i, j}$. Proposition 1 ensures that this is a legitimate definition of an aB-tree, as the value of a leaf is a function of its array element, and the value of an internal node is a function of the values of its $B$ children.

The query algorithm maintains a MTF stack $S$, which is initialized to the identity stack $S_{0}$ at the beginning of the array. Let $i \in[n]$ be the query index. The algorithm traverses down the tree, updating $S$ at each level. It maintains the invariant that whenever it visits a node $v$ whose sub-tree encompasses $m[j+1: k]$, it updates $S$ to the true stack $S_{j}$ just before the beginning of $m[j+1: k]$.
We describe how to maintain this invariant recursively. The base case is the root (at depth $d=0$ ) whose subtree contains the entire array $m$. So, the query algorithm initializes $S$ to $S_{0}$, the true initial MTF stack. Now, let $v$ be a node at depth $d$ whose sub-tree $T_{v}$ encompasses $m[j+1: k]$. Suppose the query algorithm has visited $v$, and $S$ is the true MTF stack $S_{j}$. By assumption, $j+1 \leq i \leq k$. Let $v_{1}, v_{2}, \cdots, v_{B}$ be the children of $v$ in order from left to right, and let $v_{\beta^{*}}$ be the child of $v$ whose sub-tree includes $i$. Then we update $S$ as follows:

$$
S \leftarrow \varphi_{\pi}\left(v_{\beta^{*}-1}\right) \circ \varphi_{\pi}\left(v_{\beta^{*}-2}\right) \circ \cdots \circ \varphi_{\pi}\left(v_{1}\right) \circ S .
$$

The above procedure explains the update rule which maintains the invariant at a node at depth $d+1$, assuming the invariant was maintained at a node at depth $d$. Thus, the proof that the invariant is maintained follows by induction on $d$.

Eventually, the algorithm reaches the leaf node corresponding to $m_{i}$. At this point, the MTF stack $S$ is the true stack $S_{i-1}$. Hence, it reports $x_{i}=S\left[m_{i}\right]$. The running time is $t=O\left(\lg _{B} n\right)$.

For the sake of simplicity, we have stated the update rule 7 purely in terms of forward compositions of permutations $\pi_{i, j}$. In practice, if $\beta^{*}>B / 2$, one can equivalently update $S$ by starting from $\varphi_{v} \circ S$ and composing the inverse permutations $\varphi_{\pi}^{-1}\left(v_{\beta}\right)$ for $\beta \geq \beta^{*}$ :

$$
S \leftarrow \varphi_{\pi}^{-1}\left(v_{\beta^{*}}\right) \circ \varphi_{\pi}^{-1}\left(v_{\beta^{*}+1}\right) \circ \cdots \circ \varphi_{\pi}^{-1}\left(v_{\beta}\right) \circ \varphi_{\pi}(v) \circ S .
$$

However, since all permutations $\varphi_{\pi}\left(v_{\beta}\right), \beta \in[B]$ are stored in a word, both update rules take $O(1)$ time, and so the query time remains unaltered. Henceforth, we will continue to state the update rules purely in terms of forward compositions.

\subsection{Extension to Rank Queries (over MTF)}

The aB-tree $\mathcal{T}^{\prime}$ above only supports "local decoding" (Dictionary) queries over $m=\operatorname{MTF}(x)$, while our application requires answering RANK queries. We now show how $\mathcal{T}^{\prime}$ can indeed be extended, via an additional simple observation, to obtain an aB-tree $\mathcal{T}$ supporting RANK queries under the MTF encoding.

Let $v$ be a node in the aB-tree $\mathcal{T}$, whose subtree $\mathcal{T}_{v}$ is built over the sub-array $m[i+1: j]$. We would like to augment $v$ with a vector $\tilde{\varphi}_{r k}(v)=\left(\tilde{\varphi}_{r k}\left(v, c_{\sigma}\right)\right)_{\sigma \in[|\Sigma|]} \in\{0,1, \cdots, n\}^{|\Sigma|}$, such that $\tilde{\varphi}_{r k}\left(v, c_{\sigma}\right)$ is the frequency of the character $c_{\sigma} \in \sum$ in $x[i+1: j]$. However, as $\mathcal{T}$ is built over $m=\operatorname{MTF}(x)$, and two occurrences of the same character $c \in \Sigma$ can be assigned distinct symbols in the MTF encoding, these augmented values are not consistent with the definition of an aB-tree.

To resolve this difficulty, we again use the fact that the permutation $\pi_{i, j}$ depends only on the sub-array $m[i+1: j]$. Recall that $S_{0}=\left(c_{1}, c_{2}, \cdots, c_{|\Sigma|}\right)$ corresponds to the identity permutation $\operatorname{Id}_{|\Sigma|}$. For a node $v$, let $\varphi_{r k}(v):=\left(\varphi_{r k}(v, \sigma)\right)_{\sigma \in[|\Sigma|]}$, where $\varphi_{r k}(v, \sigma)$ is the frequency of $c_{\sigma}$ in the sub-array rooted at $v$, assuming the MTF stack at the beginning of this sub-array is $S_{0}$. For a leaf node $v$ at $i \in[n]$, we have $\varphi_{r k}(v, \sigma)=1$ if $m_{i}=\sigma-1$, and 0 otherwise.

Now, let $v$ be an internal node with children $v_{1}, v_{2}, \cdots, v_{B}$. Fix a character $c_{\sigma} \in \Sigma$. In general, the MTF stack at the beginning of the sub-array rooted at $\mathcal{T}_{v}$ will be different from the MTF stack at the beginning of the sub-array $\mathcal{T}_{v_{\beta}}$ rooted at each child $v_{\beta}, \beta>1$. So, in order to express $\varphi_{r k}(v, \sigma)$ in terms of the values of its children, we need to add the entry of the vector $\varphi_{r k}\left(v_{\beta}\right)$ which corresponds to $c_{\sigma}$, 
for each $\beta \in[B]$. We do this using the permutations $\varphi_{\pi}\left(v_{\beta}\right), \beta \in[B]$. For $\beta \in[B]$, the true MTF stack at the beginning of the sub-array rooted at $v_{\beta}$, assuming the MTF stack at the beginning of the subarray rooted at $v$ is $S_{0}$, is given by Equation 7 . So, we have

$$
\varphi_{r k}(v, \sigma)=\sum_{\beta=1}^{B} \varphi_{r k}\left(v_{\beta}, \varphi_{\pi}\left(v_{\beta-1}\right) \circ \varphi_{\pi}\left(v_{\beta-2}\right) \circ \cdots \circ \varphi_{\pi}\left(v_{1}\right)(\sigma)\right)
$$

Let $\Phi_{r k}=\{0,1, \cdots, n\}^{|\Sigma|}$. We augment each node $v$ with $\varphi_{r k}(v) \in$ $\Phi_{r k}$. As we also encode the permutation $\varphi_{\pi}(v)$, the value at each internal node is a function of the values of its children, and hence this is a legitimate aB-tree.

The query algorithm, given $\left(c_{\sigma}, i\right) \in \sum \times[n]$, initializes a rank counter $r k=0$, and traverses the same root-to-leaf path as before. Fix an internal node $v$, with children $v_{1}, v_{2}, \cdots, v_{B}$, in its path. Let $\beta^{*} \in[B]$ be such that the sub-array rooted at $v_{\beta^{*}}$ contains the index $i$. The algorithm updates $r k$ as follows:

$$
r k \leftarrow r k+\sum_{\beta=1}^{\beta^{*}-1} \varphi_{r k}\left(v_{\beta}, \varphi_{\pi}\left(v_{\beta-1}\right) \circ \varphi_{\pi}\left(v_{\beta-2}\right) \circ \cdots \circ \varphi_{\pi}\left(v_{1}\right)(\sigma)\right)
$$

Then it recurses to $v_{\beta^{*}}$ and performs this step until it reaches the leaf and returns $r k_{x}\left(c_{\sigma}, i\right)$.

\subsection{Compressing the MTF aB-tree}

We now describe how to compress the aB-tree $\mathcal{T}$ defined above, using Theorem 5, to support RANK (and hence DictionARY) queries under the MTF (followed by arithmetic) encoding, with respect to the desired space bound $H_{0}(\operatorname{MTF}(x))$. Let $O(t)$ be the desired query time. Choose $B \geq 2$ such that $B \lg B=\frac{\varepsilon \lg n}{\max (t|\Sigma|, \lg \lg n)}$ for some small $\varepsilon>0$. Let $r=B^{t}$. We divide $m$ into $n / r$ sub-arrays $A_{1}, A_{2}, \cdots, A_{n / r}$ of size $r$ and build the aB-tree $\mathcal{T}$ over each subarray. So, we can support DictionARY and RANK queries within each $A_{j}$ in time $O\left(\lg _{B} r\right)=O(t)$.

For each $j \in[n / r]$, we store the true MTF stack at the beginning of the sub-array $A_{j}$, the frequency of each character $c \in \Sigma$ in the prefix $x[1:(j-1) r]$, and its index in memory.

Given a Dictionary query with index $i \in[n]$, the query algorithm determines the sub-array $A_{j}(j=\lceil i / r\rceil)$ containing $i$, initializes $S$ to the MTF stack $S_{(j-1) r}$ just before $A_{j}$, and performs the query algorithm described in Section 4.2 on the aB-tree over $A_{j}$, with query index $i-(j-1) r$.

Similarly, given a RANK query $\left(c_{\sigma}, i\right) \in \Sigma \times[n]$, the query algorithm determines the sub-array $A_{j}$ containing $i$, reads $r^{\prime}:=$ $r k_{x}\left(c_{\sigma},(j-1) r\right)$, the rank of $c_{\sigma}$ in the prefix $x[1:(j-1) r]$, and builds the permutation $\pi^{*}=\pi_{0,(j-1) r}$ corresponding to the MTF stack $S_{(j-1) r}$. Then, it performs the query algorithm described in Section 4.3 on the aB-tree over $A_{j}$, with query $\left(c_{\pi^{*}(\sigma)}, i-(j-1) r\right) \in \Sigma \times[r]$. Finally, it adds $r^{\prime}$ to this answer and returns the sum.

For a MTF character $\sigma \in\{0,1, \cdots,|\Sigma|-1\}$, let $f_{\sigma}$ be the frequency of $\sigma$ in $m$. Following [32], we define a measure of "entropy per character". For $\sigma \in\{0,1, \cdots,|\Sigma|-1\}$, we encode each occurrence of $\sigma$ in $m$ using $\lg \frac{n}{f_{\sigma}}$ bits, rounded up to the nearest multiple of $1 / r$. We impose a zeroth-order entropy constraint by augmenting each node $v$ with an additional value $\varphi_{0}(v)$, which is the sum of the entropy (suitably discretized, as described above) of the symbols in its subtree. ${ }^{15}$ We have

$H_{0}(m)=\sum_{\sigma=0}^{|\Sigma|-1} f_{\sigma} \lg \frac{n}{f_{\sigma}}=\sum_{i=1}^{n} \lg \frac{n}{f_{m_{i}}}=\sum_{j=1}^{n / r} \sum_{i \in A_{j}} \lg \frac{n}{f_{m_{i}}}=\sum_{j=1}^{n / r} H_{0}\left(A_{j}\right)$,

where $H_{0}\left(A_{j}\right)$ is the sum of entropy of the symbols in $A_{j}$. Note that the assigned entropy $\lg \frac{n}{f_{\sigma}}$ of each occurrence of a character $\sigma$ is a function of its frequency in the entire array $m$ (not in $A_{j}$ ).

Thus, for each node $v$, we encode the vector of values $\varphi(v)=$ $\left(\varphi_{\pi}(v), \varphi_{r k}(v), \varphi_{0}(v)\right)$. Now, for a given value of $\varphi=\left(\varphi_{\pi}, \varphi_{r k}, \varphi_{0}\right)$, the number of arrays $A$ of length $r$ with $H_{0}(A)=\varphi_{0}$ is at most $2^{\varphi_{0}}$ by a packing argument. So, we have $\mathcal{N}(r, \varphi) \leq \mathcal{N}\left(r, \varphi_{0}\right) \leq 2^{\varphi_{0}}$, and hence we can apply Theorem 5 to store an aB-tree of size $r$, having value $\varphi=\left(\varphi_{\pi}, \varphi_{r k}, \varphi_{0}\right)$ at the root, using $\varphi_{0}+2$ bits. Summing this space bound over all $n / r$ sub-arrays $A_{j}$, we get that the space required to store the aB-trees is at most $\sum_{j=1}^{n / r}\left(H_{0}\left(A_{j}\right)+2\right)=$ $H_{0}(m)+2 n / r$ bits.

The additional space required to store the true MTF stack and the rank of each character $c \in \Sigma$ at the beginning of each subarray $A_{j}, j \in[n / r]$, is at most $\frac{n}{r}(|\Sigma| \lg n+|\Sigma| \lg |\Sigma|)$. It is straightforward to show that the space required for the look-up tables is $O\left(n^{\varepsilon}\right)$, which is negligible for small enough $\varepsilon>0$. We defer the detailed analysis to the full version[38]. This concludes the proof of Theorem 6 .

\section{RANK DATA STRUCTURE OVER RLX}

In this section, we prove Theorem 3.1, which is restated below:

THEOREM 3.1. There exists a small constant $\delta>0$ such that for any $x \in \Sigma^{n}$ and $t^{\prime} \leq \delta \lg n$, there is a succinct data structure $\mathcal{D}_{r k}$ that supports RANK queries on $L=\mathrm{BWT}(x)$ in time $O\left(t^{\prime}\right)$, using at most $|\operatorname{RLX}(L)|+n \lg n / 2^{t^{\prime}}+n^{1-\Omega(1)}$ bits of space, in the $w=\Theta(\lg n)$ word-RAM model.

Setup and Notation. Recall that $L=\operatorname{BWT}(x) \in \Sigma^{n}$. Let $m=$ $\operatorname{MTF}(L)$. Then $m$ is a string of length $n$ over the MTF alphabet $\{0,1,2, \cdots,|\Sigma|-1\}$ (boldface symbols indicate MTF characters). Let $\bar{m}$ be the string obtained from $m$ by replacing each run of 0 's with a single character which represents its length. Thus, $\bar{m}$ is a string of length $\bar{N} \leq n$ over the expanded alphabet $\bar{\Sigma}:=[|\Sigma|-1] \cup[n]$, where $[|\Sigma|-1]:=\{1,2, \cdots,|\Sigma|-1\}$. The information-theoretic minimum space required to encode $\bar{m}$ using a zeroth order prefix-free code is $H_{0}(\bar{m})=\sum_{\sigma \in \bar{\Sigma}} f_{\sigma} \lg \frac{\bar{N}}{f_{\sigma}}$, where $f_{\sigma}$ is the frequency of $\sigma$ in $\bar{m}$, for all $\sigma \in \bar{\Sigma}$. Consider any code which converts $x$ to $m=\operatorname{MTF}(L)$ using BWT followed by MTF encoding, and then compresses $m$ using Run-length Encoding of 0-runs followed by prefix-free coding over the expanded alphabet $\bar{\Sigma}$. This code requires at least $H_{0}(\bar{m})$ bits of space. In particular, we have $|\operatorname{RLX}(L)| \geq H_{0}(\bar{m})$ by definition.

We will build an aB-tree over a slightly modified encoding of $\bar{m}$, which is similar to the one in Section 4 but is succinct with respect to $H_{0}(\bar{m})$ (and hence with respect to $|\mathrm{RLX}(L)|$ ).

Let $\varepsilon \in(0,1)$ be a small constant. We divide each run of 0 's of length $\ell_{j}>n^{\varepsilon}$ in $m$ into $\left\lceil\frac{\ell_{j}}{n^{\varepsilon}}\right\rceil$ runs of length at most $n^{\varepsilon}$ each. We then replace each run of 0 's by a single character which represents its length. Thus, we get a new string $m^{\prime}$ of length $N \leq n$ over

\footnotetext{
${ }^{15}$ This discretization is done to bound the number of possible values of $\varphi_{0}$, which is required to bound the space required for the look-up tables.
} 
the alphabet $\Sigma^{\prime}:=[|\Sigma|-1] \cup\left[n^{\varepsilon}\right]$. This is done to minimize the space required for the additional look-up tables accompanying the aB-trees which is defined later. The following lemma ensures that this step increases the space usage of the aB-trees by at most an $\tilde{O}\left(n^{1-\varepsilon}\right)$ additive term.

Lemma 2. Let $\bar{m}, m^{\prime}$ be as defined above. Then

$$
H_{0}\left(m^{\prime}\right) \leq H_{0}(\bar{m})+O\left(n^{1-\varepsilon} \lg n\right) .
$$

Intuitively, this lemma holds because the process of division of large runs introduces at most $n^{1-\varepsilon}$ additional symbols in $m^{\prime}$ as compared to $\bar{m}$. Moreover, the relative frequency of any character $\sigma \in \Sigma^{\prime}$ only changes slightly, which allows us to bound the difference in the contribution of $\sigma$ to $H_{0}(\bar{m})$ and $H_{0}\left(m^{\prime}\right)$. The final step is a simple application of the log sum inequality. We defer the formal proof to the full version [38].

\subsection{Succinct aB-tree over $m^{\prime}$}

Fix the branching factor $B \geq 2$ to be constant, and let $O\left(t^{\prime}\right)$ be the desired query time. Let $r=B^{t^{\prime}}$. We divide $m^{\prime}$ into $N / r$ sub-arrays $A_{1}^{\prime}, A_{2}^{\prime}, \cdots A_{N / r}^{\prime}$ of length $r$, and build an aB-tree over each subarray. We augment each node $v$ with a value $\varphi(v)=\left(\varphi_{\pi}(v), \varphi_{r k}(v), \varphi_{0}(v)\right)$, where $\varphi_{\pi}(v) \in \mathcal{S}_{|\Sigma|}, \varphi_{r k}(v)=\left(\varphi_{r k}(v, \sigma)\right)_{\sigma \in[|\Sigma|]} \in\left\{0,1, \cdots, n^{\varepsilon} r\right\}|\Sigma|$, and $\varphi_{0}(v) \in[0, r \lg N]$. These augmented values have the same meaning as in Section 4 . We define these values formally below.

For each node $v$, we would like $\varphi_{\pi}(v) \in \mathcal{S}_{|\Sigma|}$ to be the permutation induced by the MTF encoding on the sub-array of $m^{\prime}$ over which the subtree $\mathcal{T}_{v}$ is built. Similarly, we would like $\varphi_{r k}(v, \sigma)$ to be the frequency of $c_{\sigma}$ in the sub-array $\mathcal{T}_{v}$ rooted at $v$, assuming the MTF stack just before $\mathcal{T}_{v}$ is $S_{0}$.

First, we define the augmented values at leaf nodes. Let $v$ be a leaf node corresponding to $m_{i}^{\prime} \in \Sigma^{\prime}$ for some $i \in[N]$. If $m_{i}^{\prime}$ is a MTF symbol, i.e., $m_{i}^{\prime} \in[|\Sigma|-1]$, then we set $\varphi_{\pi}(v)$ and $\varphi_{r k}(v)$ exactly as defined in Section 4. In particular, we define $\varphi_{r k}\left(v, \sigma^{*}\right)=1$ for $\sigma^{*}=m_{i}^{\prime}+1$, and $\varphi_{r k}(v, \sigma)=0$ for all $\sigma \neq \sigma^{*}$. If $m_{i}^{\prime}$ corresponds to a run of 0 's of length $\ell_{j}$ in $\operatorname{MTF}(L)$, then we define $\varphi_{\pi}(v)=\mathbf{I d}_{|\Sigma|}$ to be the identity permutation, $\varphi_{r k}(v, 1)=\ell_{j}$, and $\varphi_{r k}(v, \sigma)=0$ for all $\sigma>1$. Here, we use the fact that the MTF stack does not change within a 0 -run.

We now define the values $\varphi_{\pi}(v)$ and $\varphi_{r k}(v)$ at each internal node $v$ recursively in terms of the values at its $B$ children $v_{1}, v_{2}, \cdots, v_{B}$, as given by Equations 6 and 8 respectively.

Finally, we specify the entropy constraint $\varphi_{0}$. Recall that for $\sigma \in \Sigma^{\prime}=[|\Sigma|-1] \cup\left[n^{\varepsilon}\right], f_{\sigma}$ denotes the frequency of $\sigma$ in $m^{\prime}$. For each $\sigma \in \Sigma^{\prime}$, we encode each occurrence of $\sigma$ in $m^{\prime}$ using $\lg \frac{N}{f_{\sigma}}$ bits, rounded up to the nearest multiple of $1 / r$. Again, following [32], we impose a zeroth-order entropy constraint by augmenting each node $v$ with $\varphi_{0}(v)$, the (suitably discretized) sum of the entropy of the symbols in its subtree.

We store the following information, for each $j \in[N / r]$ :

- The MTF stack $S_{(j-1) r}$ at the beginning of sub-array $A_{j}^{\prime}$.

- The frequency of each character $c \in \sum$ in the prefix $m^{\prime}[1$ : $(j-1) r]=\left(A_{1}^{\prime}, \cdots, A_{j-1}^{\prime}\right)$.

- The index $i_{j} \in[n]$ of the character in $m$ corresponding to the first character $m_{(j-1) r+1}^{\prime}$ of $A_{j}^{\prime}$ (if $m_{(j-1) r+1}^{\prime}$ represents a run in $m$, we store the starting index of the run). Let $T=$ $\left\{i_{j} \in[n] \mid j \in[N / r]\right\}$ be the set of indices.

We also store the map $h: T \rightarrow[N / r]$, given by $h\left(i_{j}\right)=j$ for all $j \in[N / r]$. Finally, we build a predecessor data structure $D_{\text {pred }}$ over $T$. As there are at most $\frac{N}{r} \leq \frac{n}{r}$ keys from a universe of size $n$, there exists a data structure which can answer predecessor queries in time $O\left(t^{\prime}\right)$ using space $\frac{n}{r} \cdot r^{\Omega\left(1 / t^{\prime}\right)} \cdot O(\lg n)=O\left(\frac{n \lg n}{B^{\Theta\left(t^{\prime}\right)}}\right)$ bits (for details, see [33]).

\subsection{Query algorithm}

Let the query be $(c, i) \in \Sigma \times[n]$.

- Compute $i^{\prime}=D_{\text {pred }}(i) \in T$, and index $j=h\left(i^{\prime}\right) \in[n / r]$ of the corresponding sub-array in $\mathrm{m}^{\prime}$.

- Define and initialize the following variables:

- An MTF stack $S$, initialized to $S_{(j-1) r}$ (the stack just before $A_{j}^{\prime}$ ), and the permutation $\pi^{*}=\pi_{0,(j-1) r}$.

- A rank counter $r k$, initialized to the frequency of $c$ in the prefix $m^{\prime}[1:(j-1) r]$.

- A partial sum counter $P S$, initialized to $i^{\prime}-1$. At any point, let $v$ be the last node visited by the query algorithm. Then $P S$ records the index in $m$ corresponding to the left-most node in the sub-array rooted at $v$ (for a run, we store its starting index).

- Start from the root node of the aB-tree built over $A_{j}^{\prime}$ and recursively perform the following for each node $v$ (with children $v_{1}, v_{2}, \cdots, v_{B}$ ) in the path (adaptively defined below), until a leaf node is reached:

- Let $\beta^{*} \in[B]$ be the largest index such that $P S+\sum_{\beta=1}^{\beta^{*}-1} \sum_{\sigma=1}^{\left|\sum\right|} \varphi_{r k}\left(v_{\beta}, c_{\sigma}\right) \leq i$. Update $S$ and $r k$ as specified by 7 and 9 respectively, with $\sigma$ replaced by $\pi^{*}(\sigma)$.

$$
\text { - Set } P S \leftarrow P S+\sum_{\beta=1}^{\beta^{*}-1} \sum_{\sigma=1}^{|\Sigma|} \varphi_{r k}\left(v_{\beta}, c_{\sigma}\right) \text {. }
$$

- Recurse to $v_{\beta^{*}}$.

- Let $m_{k}^{\prime}$ be the character at the leaf node. If $m_{k}^{\prime}$ represents a run of 0 's, set $c^{\prime}=S[1]$, the character at the top of the stack $S$. Otherwise, set $c^{\prime}=S\left[m_{k}^{\prime}+1\right]$.

- If $c^{\prime}=c$, set $r k \leftarrow r k+(i-P S)$.

- Return $r k$.

Now we analyze the query time. The initial predecessor query and computation of sub-array index $j$ requires $O\left(t^{\prime}\right)$ time. Then, the algorithm spends $O(1)$ time per node in the aB-tree, which has height $t^{\prime}$. Hence, the overall query time is $O\left(t^{\prime}\right)$.

\subsection{Space Analysis}

Recall that we encoded an approximation of zeroth-order entropy constraint $\varphi_{0}$ as an augmented value in the aB-tree. Using Lemma 2 and arguments similar to those in Section 4.4, we have that the aBtrees occupy at most $H_{0}\left(m^{\prime}\right)+\frac{2 N}{r} \leq H_{0}(\bar{m})+\frac{2 n}{r}+\tilde{O}\left(n^{1-\varepsilon}\right)$ bits. The additional data structures require $O(n \lg n \cdot|\Sigma| / r)$ bits of space. We can show that the look-up tables require space $O\left(n^{\varepsilon+\delta}\right)$, which is negligible for small enough $\varepsilon, \delta>0$. We defer the detailed analysis to the full version[38]. This concludes the proof of Theorem 3.1. 


\section{REFERENCES}

[1] Donald Adjeroh, Timothy Bell, and Amar Mukherjee. 2008. The Burrows-Wheeler Transform: Data Compression, Suffix Arrays, and Pattern Matching (1 ed.). Springer Publishing Company, Incorporated

[2] Rudolf Ahlswede. 1982. An elementary proof of the strong converse theorem for the multiple-access channel. 7. Combinatorics, Information and System Sciences (1982).

[3] Jon Louis Bentley, Daniel D. Sleator, Robert E. Tarjan, and Victor K. Wei. 1986. A Locally Adaptive Data Compression Scheme. Commun. ACM 29, 4 (April 1986), 320-330. https://doi.org/10.1145/5684.5688

[4] Philip Bille, Patrick Hagge Cording, Inge Li GNørtz, Benjamin Sach, Hjalte Wedel VildhNøj, and SNøren Vind. [n. d.]. Fingerprints in Compressed Strings.

[5] Andrej Brodnik and J. Ian Munro. 1999. Membership in Constant Time and Almost-Minimum Space. SIAM 7. Comput. 28, 5 (May 1999), 1627-1640. https: //doi.org/10.1137/S0097539795294165

[6] M. Burrows and D. J. Wheeler. 1994. A block-sorting lossless data compression algorithm. Technical Report 124. Digital Equipment Corporation.

[7] Yevgeniy Dodis, Mihai Patrascu, and Mikkel Thorup. 2010. Changing Base Without Losing Space. In Proceedings of the Forty-second ACM Symposium on Theory of Computing (STOC '10). ACM, New York, NY, USA, 593-602. https: //doi.org/10.1145/1806689.1806771

[8] Akashnil Dutta, Reut Levi, Dana Ron, and Ronitt Rubinfeld. 2013. A Simple Online Competitive Adaptation of Lempel-Ziv Compression with Efficient Random Access Support. In Proceedings of the 2013 Data Compression Conference (DCC '13). IEEE Computer Society, Washington, DC, USA, 113-122. https://doi.org/10 1109/DCC.2013.19

[9] Michelle Effros, Karthik Visweswariah, Sanjeev R. Kulkarni, and Sergio Verdu. 2002. Universal Lossless Source Coding With the Burrows Wheeler Transform. IEEE TRANSACTIONS ON INFORMATION THEORY 48, 5 (2002), 1061-1081.

[10] Martin Farach and Mikkel Thorup. 1995. String Matching in Lempel-Ziv Compressed Strings. In Proceedings of the Twenty-seventh Annual ACM Symposium on Theory of Computing (STOC '95). ACM, New York, NY, USA, 703-712. https://doi.org/10.1145/225058.225288

[11] Paolo Ferragina, Raffaele Giancarlo, and Giovanni Manzini. 2009. The Myriad Virtues of Wavelet Trees. Inf. Comput. 207, 8 (Aug. 2009), 849-866. https: //doi.org/10.1016/j.ic.2008.12.010

[12] Paolo Ferragina, Raffaele Giancarlo, Giovanni Manzini, and Marinella Sciortino. 2005. Boosting Textual Compression in Optimal Linear Time. 7. ACM 52, 4 (July 2005), 688-713. https://doi.org/10.1145/1082036.1082043

[13] Paolo Ferragina and Giovanni Manzini. 2005. Indexing Compressed Text. 7. ACM 52, 4 (July 2005), 552-581. https://doi.org/10.1145/1082036.1082039

[14] Travis Gagie and Giovanni Manzini. 2010. Move-to-Front, Distance Coding, and Inversion Frequencies revisited. Theoretical Computer Science 411, 31 (2010), 2925 - 2944. https://doi.org/10.1016/j.tcs.2010.04.024

[15] Travis Gagie, Gonzalo Navarro, and Nicola Prezza. 2018. Optimal-time Text Indexing in BWT-runs Bounded Space. In Proceedings of the Twenty-Ninth Annual ACM-SIAM Symposium on Discrete Algorithms (SODA '18). Society for Industrial and Applied Mathematics, Philadelphia, PA, USA, 1459-1477. http://dl.acm.org/ citation.cfm?id $=3174304.3175401$

[16] Anna Gál and Peter Bro Miltersen. 2003. The Cell Probe Complexity of Succinct Data Structures. In In Automata, Languages and Programming, 30th International Colloquium (ICALP 2003. Springer-Verlag, 332-344.

[17] Alexander Golynski. 2009. Cell probe lower bounds for succinct data structures. In Proceedings of the Twentieth Annual ACM-SIAM Symposium on Discrete Algorithms, SODA 2009, New York, NY, USA, fanuary 4-6, 2009. 625-634. http://dl.acm.org/ citation.cfm?id=1496770.1496839

[18] Alexander Golynski, Roberto Grossi, Ankur Gupta, Rajeev Raman, and S. Srinivasa Rao. 2007. On the Size of Succinct Indices. In Algorithms - ESA 2007, 15th Annual European Symposium, Eilat, Israel, October 8-10, 2007, Proceedings. 371-382. https://doi.org/10.1007/978-3-540-75520-3 34

[19] Alexander Golynski, Rajeev Raman, and S. Srinivasa Rao. 2008. On the Redundancy of Succinct Data Structures. In Proceedings of the 11th Scandinavian Workshop on Algorithm Theory (SWAT '08). Springer-Verlag, Berlin, Heidelberg, 148-159. https://doi.org/10.1007/978-3-540-69903-3 15

[20] Haim Kaplan, Shir Landau, and Elad Verbin. 2007. A Simpler Analysis of BurrowsWheeler-based Compression. Theor. Comput. Sci. 387, 3 (Nov. 2007), 220-235. https://doi.org/10.1016/j.tcs.2007.07.020

[21] Haim Kaplan and Elad Verbin. 2007. Most Burrows-wheeler Based Compressors Are Not Optimal. In Proceedings of the 18th Annual Conference on Combinatorial Pattern Matching (CPM'07). Springer-Verlag, Berlin, Heidelberg, 107-118. http: //dl.acm.org/citation.cfm?id=2394373.2394390
[22] S. Rao Kosaraju and Giovanni Manzini. 1999. Compression of Low Entropy Strings with Lempel-Ziv Algorithms. SIAM 7. Comput. 29, 3 (Dec. 1999), 893-911. https://doi.org/10.1137/S0097539797331105

[23] Udi Manber and Gene Myers. 1990. Suffix Arrays: A New Method for On-line String Searches. In Proceedings of the First Annual ACM-SIAM Symposium on Discrete Algorithms (SODA '90). Society for Industrial and Applied Mathematics, Philadelphia, PA, USA, 319-327. http://dl.acm.org/citation.cfm?id=320176. 320218

[24] Giovanni Manzini. 2001. An Analysis of the Burrows-Wheeler Transform. 7. ACM 48, 3 (May 2001), 407-430. https://doi.org/10.1145/382780.382782

[25] Giovanni Manzini and Paolo Ferragina. 2004. Engineering a Lightweight Suffix Array Construction Algorithm. Algorithmica 40, 1 (June 2004), 33-50. https: //doi.org/10.1007/s00453-004-1094-1

[26] Edward M. McCreight. 1976. A Space-Economical Suffix Tree Construction Algorithm. F. ACM 23, 2 (April 1976), 262-272. https://doi.org/10.1145/321941. 321946

[27] Peter Bro Miltersen. 2005. Lower Bounds on the Size of Selection and Rank Indexes. In Proceedings of the Sixteenth Annual ACM-SIAM Symposium on Discrete Algorithms (SODA '05). Society for Industrial and Applied Mathematics, Philadelphia, PA, USA, 11-12. http://dl.acm.org/citation.cfm?id=1070432.1070435

[28] Alistair Moffat. 1990. Implementing the PPM Data Compression Scheme.

[29] J. Ian Munro, Rajeev Raman, Venkatesh Raman, and Srinivasa Rao S. 2012. Succinct Representations of Permutations and Functions. Theor. Comput. Sci. 438 (June 2012), 74-88. https://doi.org/10.1016/j.tcs.2012.03.005

[30] Gonzalo Navarro and Veli Mäkinen. 2007. Compressed Full-text Indexes. ACM Comput. Surv. 39, 1, Article 2 (April 2007). https://doi.org/10.1145/1216370. 1216372

[31] Rasmus Pagh. 2002. Low Redundancy in Static Dictionaries with Constant Query Time. SIAM 7. Comput. 31, 2 (Feb. 2002), 353-363. https://doi.org/10.1137/ S0097539700369909

[32] Mihai Pătrașcu. 2008. Succincter. In Proceedings of the 2008 49th Annual IEEE Symposium on Foundations of Computer Science (FOCS '08). IEEE Computer Society, Washington, DC, USA, 305-313. https://doi.org/10.1109/FOCS.2008.83

[33] Mihai Pătrașcu and Mikkel Thorup. 2006. Time-space Trade-offs for Predecessor Search. In Proceedings of the Thirty-eighth Annual ACM Symposium on Theory of Computing (STOC '06). ACM, New York, NY, USA, 232-240. https://doi.org/10. 1145/1132516.1132551

[34] Mihai Pătrașcu and Emanuele Viola. 2010. Cell-probe Lower Bounds for Succinct Partial Sums. In Proceedings of the Twenty-first Annual ACM-SIAM Symposium on Discrete Algorithms (SODA '10). Society for Industrial and Applied Mathematics, Philadelphia, PA, USA, 117-122. http://dl.acm.org/citation.cfm?id=1873601. 1873612

[35] Kunihiko Sadakane and Roberto Grossi. 2006. Squeezing Succinct Data Structures into Entropy Bounds. In Proceedings of the Seventeenth Annual ACM-SIAM Symposium on Discrete Algorithm (SODA '06). Society for Industrial and Applied Mathematics, Philadelphia, PA, USA, 1230-1239. http://dl.acm.org/citation.cfm? id $=1109557.1109693$

[36] Julian Seward. [n. d.]. Bzip2. www.bzip.org

[37] Jared T. Simpson and Richard Durbin. 2010. Efficient construction of an assembly string graph using the FM-index. Bioinformatics [ISMB] 26, 12 (2010), 367373. http://dblp.uni-trier.de/db/journals/bioinformatics/bioinformatics26.html\# SimpsonD10

[38] Sandip Sinha and Omri Weinstein. 2018. Local Decodability of the BurrowsWheeler Transform. arXiv: 1808.03978

[39] Emanuele Viola. 2009. Cell-Probe Lower Bounds for Prefix Sums. Electronic Colloquium on Computational Complexity (ECCC) 16 (2009), $54 . \quad$ http://eccc. hpi-web.de/report/2009/054

[40] Emanuele Viola. 2012. Bit-Probe Lower Bounds for Succinct Data Structures. SIAM f. Comput. 41, 6 (2012), 1593-1604. https://doi.org/10.1137/090766619 arXiv:https://doi.org/10.1137/090766619

[41] Emanuele Viola. 2017. A sampling lower bound for permutations. Electronic Colloquium on Computational Complexity (ECCC) 24 (2017), 166. https://eccc. weizmann.ac.il/report/2017/166

[42] Emanuele Viola. 2018. Sampling lower bounds: boolean average-case and permutations. Electronic Colloquium on Computational Complexity (ECCC) 25 (2018), 60. https://eccc.weizmann.ac.il/report/2018/060

[43] J. Ziv and A. Lempel. 2006. Compression of Individual Sequences via Variable-rate Coding. IEEE Trans. Inf. Theor. 24, 5 (Sept. 2006), 530-536. https://doi.org/10. 1109/TIT.1978.1055934

[44] J. Ziv and A. Lempel. 2006. A Universal Algorithm for Sequential Data Compression. IEEE Trans. Inf. Theor. 23, 3 (Sept. 2006), 337-343. https://doi.org/10.1109/ TIT.1977.1055714 